\title{
Análisis crítico del concepto "aprendizaje ubicuo" a través de la Cartografía Conceptual
}

\section{Critical analysis of the concept "ubiquitous learning" through Conceptual Cartography}

\author{
Belén Velázquez Gatica \\ Universidad Autónoma de Querétaro. Santiago de Querétaro, México \\ bvelazquez829@alumnos.uaq.mx \\ Rocío Edith López Martínez \\ Universidad Autónoma de Querétaro. Santiago de Querátaro, México \\ rocio.edith.lopez@uaq.mx
}

\begin{abstract}
Resumen
El concepto "aprendizaje ubicuo" se ha criticado por su carácter tecnocéntrico, debido a que enfatiza los aspectos tecnológicos por encima de los educativos. No obstante, una vez que el aprendizaje ubicuo evolucione hacia un concepto educativo, sus aportes podrán alcanzar mayor relevancia y pertinencia en el marco de la ubicuidad y sus aplicaciones en el aprendizaje. Por tal motivo, el objetivo del presente trabajo fue realizar una revisión documental sobre el término aprendizaje ubicuo, para elaborar un análisis crítico que apoye en su desarrollo. Para ello, se utilizó el método de Cartografía Conceptual, a través de un procedimiento en cuatro fases: 1) Búsqueda de documentos pertinentes a la investigación; 2) Definición de criterios de inclusión y exclusión de documentos; 3) Análisis de datos a partir de los ejes de la cartografía conceptual (Noción, Caracterización, Categorización, Diferenciación, Clasificación, Ejemplificación y Vinculación); 4) Interpretación de resultados. El resultado principal sugiere que el aprendizaje ubicuo maduró conceptualmente, debido a la incorporación de dos enfoques: el aprendizaje continuo y el personalizado. No obstante, estos enfoques se basan en ideales del aprendizaje, por lo que es necesario precisar conceptos que apoyen la perspectiva del aprendizaje ubicuo a la hora de establecer prácticas educativas concretas.
\end{abstract}

\section{Palabras clave}

Aprendizaje ubicuo, Cartografía Conceptual, Ubicuidad tecnológica, Aprendizaje continuo, Contexto.

\begin{abstract}
The concept of "ubiquitous learning" has been criticized due to its technocentric nature, which emphasizes technological aspects over educational ones. However, once ubiquitous learning evolves into an educational concept, its contributions may become more relevant and pertinent in the context of ubiquity and its applications in learning. For this reason, the objective of this work was to carry out a documentary review on the term ubiquitous learning, to make a critical analysis that supports its development. The Conceptual Cartography method was used, through a four-phase procedure: 1) Search for documents relevant to the research; 2) Definition of inclusion and exclusion criteria for documents; 3) Data analysis with analytical categories (Notion, Characterization, Categorization, Differentiation, Classification, Exemplification and Linkage); 4) Interpretation of results. The main result suggests that ubiquitous learning matured conceptually, due to the incorporation of two approaches: continuous and personalized learning. However, these approaches are
\end{abstract}


based on learning ideals, so it is necessary to specify concepts that support the ubiquitous learning perspective when establishing concrete educational practices.

\section{Keywords:}

Ubiquitous learning, Conceptual Cartography, Technological ubiquity, Continuous learning, Context.

\section{Introducción}

A medida que los dispositivos móviles estuvieron disponibles para un gran número de personas, y que se configuró un escenario de convergencia tecnológica cotidiana sin precedentes, también aumentaron las posibilidades de su aplicación en distintos contextos, entre ellos, el educativo (E. Fernández \& Anguita, 2015; González-Sanmamed et al., 2018; Hermann, 2013; Sevillano-García et al., 2016). La mayor capacidad de aprendizaje que proporcionaron los dispositivos móviles sobre otras tecnologías, es que no estaban fijos espacialmente, sino que se podían usar en sitios formales e informales y de manera individual o involucrar a otros en el aprendizaje, gracias a la conectividad (Bernacki, Crompton, et al., 2020).

Esta portabilidad permitió, además, la aparición de nuevas pedagogías y vías de investigación como el aprendizaje ubicuo o u-learning (Bernacki, Crompton, et al., 2020). En primera instancia, se reconoce la ubicuidad en el aprendizaje cuando el individuo que aprende, puede estar virtualmente presente en muchos lugares a la vez (García, 2017). Asimismo, diversos autores señalaron que la mayor característica del aprendizaje ubicuo está en la deslocalización y en la intemporalidad en los procesos de educación y de enseñanza (M. Fernández, 2013; Sevillano-García et al., 2016), puesto que el u-learning no solo aprovecha los diferentes dispositivos y modalidades para sus propósitos (Hermann, 2013), sino que también optimiza diferentes tiempos, siendo así contrario a la visión lineal del aprendizaje.

La ubicuidad del aprendizaje es una posibilidad actual que se da por hecho, de manera que, acceder al aprendizaje en cualquier lugar y momento se está convirtiendo en una necesidad (El Guabassi et al., 2018) y en un motivo de exclusión si no se practica. No obstante, esta visión del aprendizaje ubicuo que descuida el aspecto pedagógico y se centra solo en las posibilidades tecnológicas, se ha criticado por su visión tecnocentrista, es decir, por colocar las funciones de la tecnología por encima de los aspectos social, cultural, filosófico y educativo (Hermann, 2013). Además de ser utópica en los países con mayores brechas digitales.

Otras críticas al respecto, señalan que lo importante de la imbricación de las tecnologías no es la diversidad de herramientas con que cuentan los profesores y los estudiantes, sino la variedad de escenarios de comunicación y de formación que se han creado, y que se caracterizan por ubicar a los estudiantes en el centro del escenario, además de sacar el aprendizaje de una concepción aislada e individual, hacia posiciones más colaborativas $\mathrm{y}$, de ampliar las fuentes de información con las que se puede interaccionar (Cabero \& Llorente, 2015). Asimismo, se enfatiza la personalización del aprendizaje a través de sistemas y estrategias apropiadas (Huang \& Chiu, 2015).

Con base en las críticas hacia el aprendizaje ubicuo, diversos autores han afirmado que se trata de un concepto aún en desarrollo, que una vez que evolucione, puede llegar a hacer aportes importantes que permitan una adecuada incorporación de la ubicuidad

Análisis crítico del concepto "aprendizaje ubicuo" a través de la Cartografía Conceptual. Belén Velázquez Gatica y Rocío Edith López Martínez. 
tecnológica al contexto educativo (Báez \& Clunie, 2019). Para ello será necesario llevar a cabo más investigación que permita entender y definir su núcleo teórico y su alcance (Casanovas \& Tomassino, 2014). Por tal motivo, el objetivo del presente trabajo fue realizar una revisión documental de la literatura especializada sobre el concepto de aprendizaje ubicuo, a través de la cartografía conceptual, con el propósito de elaborar un análisis crítico que apoye en el desarrollo de dicho concepto.

\section{Método}

Para cumplir el objetivo de investigación se realizó una cartografía conceptual, un método de investigación documental basado en el pensamiento complejo, que consiste en estudiar un concepto con profundidad, para sistematizar la información existente sobre tal concepto, así como construir los aspectos faltantes tomando como base información especializada, a fin de comprenderlo y comunicarlo con claridad en la comunidad académica (Tobón et al., 2015). La cartografía conceptual no se basa solo en apoyar la gestión del conocimiento desde un análisis conceptual, sino también teórico y práctico a partir de aspectos verbales, no verbales y espaciales para dar cuenta de sus relaciones y organización, aspectos que posibilitan el proceso de comprensión (Ortega-Carbajal et al., 2015; Tobón-Tobón, 2004).

Desde la cartografía conceptual se entiende que los conceptos son constructos mentales que ayudan a comprender objetos de la realidad interior del hombre (subjetiva) y del exterior (objetiva), y tienen una clasificación, caracterización, diferenciación, composición, atributos y relaciones, de tal forma que son tejidos relacionados y ello precisa una aproximación cartográfica para construirlos, comunicarlos y comprenderlos (Tobón-Tobón, 2004). En el marco de la cartografía conceptual los elementos que ayudan a comprender los conceptos desde la complejidad son (Tobón-Tobón, 2004, pp. 9-10):

- La construcción de conceptos es un proceso permanente, nunca acabado, acorde a los cambios sociales y culturales que influyen en el lenguaje de las ciencias;

- En la elaboración de conceptos es necesaria la transdisciplinariedad, lo cual implica que la comprensión de un término científico en toda su complejidad trasciende los límites estrechos de una sola disciplina, siendo necesaria la psicolingüística, la sociología, la filología, etc.

- La construcción del término y su comunicación se hacen entretejiendo relaciones entre diferentes aspectos que le aportan sentido y le dan precisión.

- La didáctica de los conceptos implica conjugar lo verbal con lo no verbal y el hacer, con el fin de que haya una adecuada comprensión, teniendo como base el apoyo en técnicas gráficas.

La cartografía conceptual propone siete ejes para construir de una forma estandarizada los conceptos (Tobón-Tobón, 2004, pp. 11-12):

- Eje nocional: Se da una aproximación al concepto estableciendo su definición corriente y el origen de la palabra o palabras de las cuales se compone.

- Eje categorial: Se describe la clase general de conceptos dentro de la cual está incluido el concepto en cuestión.

- Eje de diferenciación: Se establecen una o varias proposiciones en las cuales se muestre la diferencia de ese concepto de otros conceptos similares.

- Eje de ejemplificación: Se describen proposiciones que ejemplifiquen el concepto con casos específicos.

Análisis crítico del concepto "aprendizaje ubicuo" a través de la Cartografía Conceptual. Belén Velázquez Gatica y Rocío Edith López Martínez. 
- Eje de caracterización: Se describen las características esenciales del concepto.

- Eje de subdivisión: Se construyen las clases en las cuales se clasifica o divide el concepto.

- Eje de vinculación: Se establecen las relaciones de ese concepto con otros que son importantes desde lo semántico o contextual.

El procedimiento de análisis crítico se desarrolló en cuatro fases a través de preguntas de investigación. Dicho procedimiento se describe a continuación:

1. Búsqueda de documentos pertinentes a la investigación. Se realizó una búsqueda sistemática de artículos de investigación en las bases de datos Redalyc, Web of Science y Science Direct, desde enero hasta febrero de 2020 y el análisis se realizó desde febrero hasta abril de 2020. Se admitieron textos publicados en español e inglés desde 1991 -año en que se utilizó por primera vez el término aprendizaje ubicuo- hasta 2020. Los filtros generales que se utilizaron en las tres bases de datos fueron: idioma (inglés y español) y año (1991-2020) y en el caso de Science Direct y Web of Science, los buscadores permitieron aplicar el filtro de tipo de artículo (artículos de investigación y artículos de revisión), mientras que se excluyeron reseñas y capítulos de libro, discusiones, editoriales, conferencias, entre otros.

2. Definición de criterios de inclusión y exclusión de los documentos obtenidos. La búsqueda en las bases de datos científicas se realizó a partir un criterio booleano con los términos: ("aprendizaje ubicuo") AND (teoría) OR (concepto) y ("ubiquitous learning") AND (theory) OR (concept). Se decidió restringir el término específico (aprendizaje ubicuo) en español y (ubiquitous learning) en inglés para evitar resultados secundarios al tema. La búsqueda se efectuó sobre el título, el resumen, las palabras clave y el documento extenso. Se obtuvo la siguiente cantidad de documentos: Science Direct, 235 artículos en inglés y 0 en español; Redalyc, 27 artículos en inglés y 60 en español; en el caso de Web of Science se obtuvo una cantidad considerable de resultados, por lo que se utilizó la opción de Relevancia y se limitó la inclusión de artículos a los primeros 100 elementos listados.

En esta fase se obtuvieron 422 documentos. Posteriormente, se identificaron los elementos duplicados y se eliminaron. Además, se realizó una revisión superficial de la pertinencia de los artículos obtenidos y se excluyeron aquellos que no eran útiles para el análisis de esta investigación. En esta etapa se conservaron 44 trabajos de Science Direct, 26 de Web of Science y 30 de Redalyc. En la última fase de selección, se efectuó una lectura minuciosa de los documentos, y se descartaron aquellos artículos a) cuyo contenido no mostraba relación directa con el aprendizaje ubicuo, b) los que a pesar de que si tenían relación con los términos de búsqueda, su texto no aportaba información correspondiente a los ejes de análisis de la cartografía conceptual. Finalmente, se admitieron 69 artículos para el análisis (25 en español y 44 en inglés).

3. Análisis de los documentos a partir de los ejes de la cartografía conceptual. El análisis de los artículos se realizó con base en los siete ejes propuestos en la cartografía conceptual para construir conceptos de una forma estandarizada:

Análisis crítico del concepto "aprendizaje ubicuo" a través de la Cartografía Conceptual. Belén Velázquez Gatica y Rocío Edith López Martínez. 
Noción, Categorización, Diferenciación, Ejemplificación, Caracterización, Subdivisión (Clasificación) y Vinculación (Tobón-Tobón, 2004). El análisis se desarrolló respondiendo las siguientes preguntas (Tobón-Tobón, 2012):

Tabla 1

Ejes de la cartografía conceptual del aprendizaje ubicuo basados en Tobón et al., (2015).

\begin{tabular}{|c|c|c|}
\hline Categoría de análisis & Pregunta de investigación & Componentes \\
\hline 1. Noción & $\begin{array}{lrr}\text { ¿Cuál es } & \text { el } & \text { origen del } \\
\text { concepto } & & \begin{array}{r}\text { aprendizaje } \\
\text { desarrollo }\end{array} \\
\text { ubicuo, } & \text { su } & \text { desarión } \\
\text { histórico y la } & \text { definición } \\
\text { actual? } & & \end{array}$ & $\begin{array}{l}\text {-Origen del aprendizaje } \\
\text { ubicuo } \\
\text {-Desarrollo histórico del } \\
\text { enfoque } \\
\text {-Definición actual }\end{array}$ \\
\hline 2. Categorización & $\begin{array}{l}\text { ¿A qué categoría o clase } \\
\text { mayor pertenece el concepto } \\
\text { aprendizaje ubicuo? }\end{array}$ & $\begin{array}{l}\text {-Clase inmediata: definición y } \\
\text { características }\end{array}$ \\
\hline 3. Caracterización & $\begin{array}{l}\text { ¿Cuáles son los elementos } \\
\text { centrales que le dan } \\
\text { identidad al aprendizaje } \\
\text { ubicuo? }\end{array}$ & $\begin{array}{l}\text {-Características claves del } \\
\text { enfoque teniendo en cuenta la } \\
\text { noción y la categorización }\end{array}$ \\
\hline 4. Diferenciación & $\begin{array}{l}\text { ¿De cuáles otros enfoques } \\
\text { cercanos se diferencia el } \\
\text { aprendizaje ubicuo? }\end{array}$ & $\begin{array}{l}\text {-Se describen los enfoques de } \\
\text { aprendizaje cercanos, se } \\
\text { definen y se diferencian }\end{array}$ \\
\hline 5. Clasificación & $\begin{array}{l}\text { ¿En qué subclases o tipos se } \\
\text { clasifica el aprendizaje } \\
\text { ubicuo? }\end{array}$ & $\begin{array}{l}\text {-Se establecen los criterios de } \\
\text { clasificación } \\
\text {-Se determinan los tipos de } \\
\text { aprendizaje ubicuo de acuerdo } \\
\text { con los criterios seleccionados }\end{array}$ \\
\hline 6. Ejemplificación & $\begin{array}{l}\text { ¿Cuál sería un ejemplo } \\
\text { relevante y pertinente de } \\
\text { aplicación del aprendizaje } \\
\text { ubicuo? }\end{array}$ & $\begin{array}{l}\text {-Se describen proposiciones } \\
\text { que ejemplifiquen el concepto } \\
\text { con casos específicos } \\
\text {-Se describe un ejemplo } \\
\text { concreto que ilustre la } \\
\text { aplicación del aprendizaje } \\
\text { ubicuo. }\end{array}$ \\
\hline 7. Vinculación & $\begin{array}{l}\text { ¿Cómo se relaciona el } \\
\text { aprendizaje ubicuo con } \\
\text { determinadas teorías, } \\
\text { procesos sociales-culturales } \\
\text { y referentes epistemológicos } \\
\text { que están por fuera de la } \\
\text { categorización? }\end{array}$ & $\begin{array}{l}\text {-Se describen uno o varios } \\
\text { enfoques o teorías diferentes a } \\
\text { la categorización que brindan } \\
\text { contribuciones a la } \\
\text { comprensión, construcción y } \\
\text { aplicación del aprendizaje } \\
\text { ubicuo. } \\
\text {-Se indican las contribuciones } \\
\text { de estos enfoques o teorías al } \\
\text { aprendizaje ubicuo }\end{array}$ \\
\hline
\end{tabular}

Fuente: elaboración propia

Análisis crítico del concepto "aprendizaje ubicuo" a través de la Cartografía Conceptual. Belén Velázquez Gatica y Rocío Edith López Martínez. 
4. Interpretación de resultados. Los resultados de esta fase se muestran en el apartado de conclusiones.

\section{Resultados}

\subsection{Noción: ¿Cuál es el origen del concepto aprendizaje ubicuo, su desarrollo histórico y la definición actual?}

Diversos autores señalaron que el origen del concepto aprendizaje ubicuo surgió de Weiser (Casanovas \& Tomassino, 2014; G. D. Chen et al., 2008; Park, 2011), quien utilizó en 1991 por primera vez, los términos computación e informática ubicua para describir las posibilidades futuras de la tecnología a través de su naturalización en todos los grupos de la sociedad. Su filosofía era contraria a la realidad virtual que trataba de introducir a la persona dentro del computador, puesto que se esperaba que las computadoras se integraran en la vida de las personas y no lo opuesto, además de cuestionar el paradigma de la computadora de escritorio (Abascal \& Moriyón, 2002).

En esta proyección sobre las tecnologías, Weiser (1991) refirió que el verdadero potencial de la informática se vería cuando estas formaran parte invisible e integral de la vida de las personas. Dicho potencial no vendría de una tecnología en especial, sino de la interacción de todas ellas y al interior de ellas, entre la suma de diversas pestañas (Weiser, 1991). La relación histórica entre aprendizaje ubicuo y computación ubicua se debe a que en los años 90's había una certeza de que las computadoras seguirían siendo el medio por excelencia para realizar diversas actividades digitales en la vida cotidiana. Es decir, el aprendizaje ubicuo surge como un concepto tecnocentrista ligado a la naturalización del uso de las computadoras para apoyar el aprendizaje diario.

Sin embargo, la convergencia mediática y el surgimiento y expansión de los dispositivos móviles crearon nuevas posibilidades (Casanovas \& Tomassino, 2014; Manolakis, 2011) que construyeron no un concepto tecnocentrista, sino un nuevo enfoque social que se caracteriza principalmente por el dominio de los tiempos y los espacios para aprender y que para ello, se vale de la ubicuidad que se obtiene de la suma de diferentes elementos: tecnologías y modalidades educativas (Huang et al., 2011), la conectividad, la democratización del aprendizaje (Ferreira \& Castilho, 2018; López, 2017), más el imaginario social del aprendizaje continuo y a lo largo de toda la vida (Cobo \& Moravec, 2011), en donde no solo se espera que el aprendiz gestione sus procesos de aprendizaje, sino que se exige una fuerte autonomía en dichos procesos.

El centro de interés en el aprendizaje ubicuo pasó de enfocarse en la naturalización de las computadoras a definirse como la capacidad de aprender en cualquier lugar y en cualquier momento a través de diferentes dispositivos digitales (Báez \& Clunie, 2019; GarcíaSánchez \& Santos-Espino, 2017; Moreno-López et al., 2017; Wen \& Zhang, 2014), definición que en la actualidad es la más aceptada. Es decir, una visión basada en el individuo y su capacidad constante y autónoma para iniciar el aprendizaje con tecnologías. Ambas nociones, el aprendizaje ubicuo como término tecnocentrista y como enfoque social regulador no han estado exentos de críticas, debido a que son, como muchos conceptos tecnológicos cuando surgen, generales, radicales o utópicos. Las problemáticas se originan a la hora de implementarlos en prácticas educativas concretas, donde se hace necesario matizar o incrementar detalles.

El contexto en el aprendizaje ubicuo es el elemento que más se ha considerado a la hora de ajustar el concepto o aumentar sus detalles (Brazuelo \& Gallego, 2014). Su

Análisis crítico del concepto "aprendizaje ubicuo" a través de la Cartografía Conceptual. Belén Velázquez Gatica y Rocío Edith López Martínez. 
incorporación responde al interés en reflexionar menos sobre los detalles técnicos de las máquinas y más sobre su uso situacional, es decir, cómo las tecnologías se integran en las actividades sociales humanas (de Sousa et al., 2014). No obstante, cuando se incrementan detalles en los conceptos sin seguir un eje analítico, las definiciones del concepto se diversifican, por lo que no hay un consenso establecido sobre lo que es el aprendizaje ubicuo. Su definición dependerá del énfasis que se le da a ciertas características tecnológicas, individuales, educativas o la combinación de ellas.

Algunos tienen una perspectiva relativamente tecnocéntrica, que trata las tecnologías ubicuas como las habilitadoras esenciales del aprendizaje continuo sin ser interrumpidos, mientras los alumnos cambian de ubicación o dispositivos (Wong \& Looi, 2011). Otros basan su definición en lo educativo y en los flujos de aprendizaje y señalan que el $u$ learning describe el universo de actividades formativas apoyadas en tecnologías, disponibles en cualquier momento, lugar y situación que devienen en un aprendizaje continuo, en tiempo real y basado en la localización contextual del aprendiente (Artopoulos \& Kozak, 2011; Cárdenas-Robledo \& Peña-Ayala, 2018; Casanovas \& Tomassino, 2014). Mientras que hay quienes ubican el aprendizaje ubicuo como una capacidad individual de participar en situaciones de aprendizaje en cualquier momento y en cualquier lugar, sin necesitar de presencia física, sino mediado por tecnologías digitales (López, 2017).

Es por ello, que es necesario analizar el concepto de aprendizaje ubicuo para distinguir qué es lo que permite en realidad que un aprendizaje se considere como tal.

\subsection{Categorización: ¿A qué categoría o clase mayor pertenece el concepto aprendizaje ubicuo?}

El concepto de aprendizaje ubicuo se originó en el marco de la informática ubicua o computación ubicua. Otras aproximaciones ubican el concepto como un tipo de aprendizaje móvil, que a su vez es un dominio del e-learning. Mientras que la influencia de enfoques como el aprendizaje continuo y el aprendizaje personalizado/adaptativo han configurado su definición más actual, y han impulsado la madurez del concepto en términos educativos, transitando de una visión tecnocentrista y generalizada del aprendizaje, hacia un enfoque que promueve el dominio del aprendizaje personalizado sobre el espacio y el tiempo.

Respecto a la primera perspectiva, el u-learning se observa como uno de los campos de aplicación de la computación ubicua (Moreno-López et al., 2017). La computación generalizada, como también se le conoce, es una línea de investigación basada en la visión de Weiser (Lucke \& Rensing, 2013), quien planteó un escenario en el que la computadora se volvería invisible e indistinguible de su entorno, de tal forma que la interacción entre humanos y computadoras no ocurriría como de costumbre, donde los individuos dedican toda su atención a la computadora sino que consistiría en interacciones pequeñas y rápidas de manera cotidiana (Wagner et al., 2014).

Debido al desarrollo de dispositivos digitales diferentes a las computadoras, se prefiere en la actualidad denominarse informática ubicua al campo que estudia la integración de las tecnologías en la vida y el entorno de las personas. Desde la informática generalizada el aprendizaje ubicuo es un tipo de aprendizaje mejorado, con un entorno inteligente y una conciencia del contexto, que apoya dinámicamente el aprendizaje mediante la comunicación con los dispositivos integrados en el entorno, mientras un estudiante se

Análisis crítico del concepto "aprendizaje ubicuo" a través de la Cartografía Conceptual. Belén Velázquez Gatica y Rocío Edith López Martínez. 
mueve con su dispositivo móvil, de tal forma que mediante las tecnologías, los usuarios se enriquecen con una gran experiencia de aprendizaje (Vinu et al., 2011).

Desde la perspectiva del $u$-learning como un tipo de aprendizaje móvil, se describe como aquel aprendizaje que permite acceder a diversos entornos, contextos y situaciones, y que puede utilizar una mayor conciencia del contexto para proporcionar la mayoría de los contenidos adaptativos para los estudiantes (Oliveira \& Horta, 2012). No obstante, hay autores que señalan que el aprendizaje ubicuo no es un tipo de m-learning, sino que se trata de un concepto más amplio que el primero o que representa su evolución (Gilman et al., 2015), debido a que el u-learning intenta aprovechar los diferentes dispositivos móviles o fijos, distintos momentos y modalidades a través de su integración para el aprendizaje (Coto et al., 2016; De la Torre et al., 2013; Huang et al., 2008; Kim et al., 2012; Marinagi et al., 2013; Moreno et al., 2016; Ramirez et al., 2017). Esta visión del aprendizaje ubicuo es resultado de la influencia de dos enfoques: el aprendizaje continuo y el aprendizaje personalizado/adaptativo.

El aprendizaje continuo aboga por aprender en cualquier momento y en cualquier lugar. Sin embargo, esto no implica que los aprendices siempre estén persiguiendo el aprendizaje, sino que se busca que se empoderen y estén estimulados para aprender cuando lo necesiten y donde lo requieran (Wong \& Looi, 2011). El aprendizaje continuo no se apoya en la tecnología como componente esencial, sino en la importancia de vincular las experiencias de los estudiantes dentro y fuera de clase, sin interrupciones. En este sentido, el aprendizaje continuo es más un hábito mental del estudiante para conseguir un flujo continuo de aprendizaje en todos los contextos, y puede o no ser mediado por la tecnología (Wong \& Looi, 2011).

En cuanto al aprendizaje personalizado/adaptativo se define como un proceso que recoge las características de individuales y las necesidades de los estudiantes, para promover actividades de aprendizaje significativas y relevantes para los estudiantes, las cuales a menudo son autoiniciadas (Xie et al., 2019). Este tipo de aprendizaje también puede apoyarse o no en las tecnologías. En el primer caso, la tecnología emite recomendaciones basadas en las preferencias de los estudiantes.

En resumen, si bien se reconoce a la informática generalizada como la categoría tecnológica del concepto de aprendizaje ubicuo, esta implica una visión tecnocentrista. Por lo que el u-learning se ha influenciado y ha integrado enfoques como el aprendizaje continuo y el aprendizaje personalizado/adaptativo. Esto ha permitido que el aprendizaje ubicuo evolucione hacia un concepto educativo. De esta forma, ambos enfoques se reconocen como categorías educativas del término.

La integración de la informática generalizada, el aprendizaje continuo y el aprendizaje personalizado/adaptativo permitieron que en el u-learning se reconociera la importancia del contexto, la motivación y los intereses del individuo que aprende y, que dichas características son variables. Esto tiene dos implicaciones. Primero, se adopta la idea de que es el individuo quien determina el uso de las tecnologías, en una situación de aprendizaje y no la tecnología en sí, como se planteaba desde la computación ubicua. Segundo, la ubicuidad en el aprendizaje dejó de observarse de arriba hacia abajo, es decir, el aprendizaje no es algo que se da de forma generalizada, en todo lugar y todo momento, sino algo que depende de una situación de aprendizaje o de un contexto específico.

Análisis crítico del concepto "aprendizaje ubicuo" a través de la Cartografía Conceptual. Belén Velázquez Gatica y Rocío Edith López Martínez. 


\subsection{Caracterización: ¿Cuáles son los elementos centrales que le dan identidad al aprendizaje ubicuo?}

El aprendizaje ubicuo se materializa en la construcción de sistemas o entornos de $u$ learning, que se conforman a partir de tres elementos esenciales anteriormente referidos: la tecnología, el aspecto pedagógico y los flujos de aprendizaje, y las características del individuo que aprende. Cada uno de estos componentes contribuye con ciertos aspectos que configuran el aprendizaje ubicuo en un sistema ideal, puesto que en ocasiones los entornos se crean enfatizando un solo elemento. Por ejemplo, es común la construcción de sistemas de aprendizaje ubicuo tecnocentristas. Cabe destacar que en la literatura especializada sobre el tema estos elementos no están desagregados, no obstante, aquí se dividen con propósitos de análisis (ver Tabla 2). Esto implica que algunas de las características puedan incluirse en dos o tres componentes, puesto que su propiedad se forma de la conjunción de elementos.

Tabla 2

Características de los entornos de aprendizaje ubicuo

\begin{tabular}{|c|c|c|}
\hline Característica & Tecnología & $\begin{array}{l}\text { Pedagogía } \\
\text { y flujos de } \\
\text { aprendizaje }\end{array}$ \\
\hline
\end{tabular}

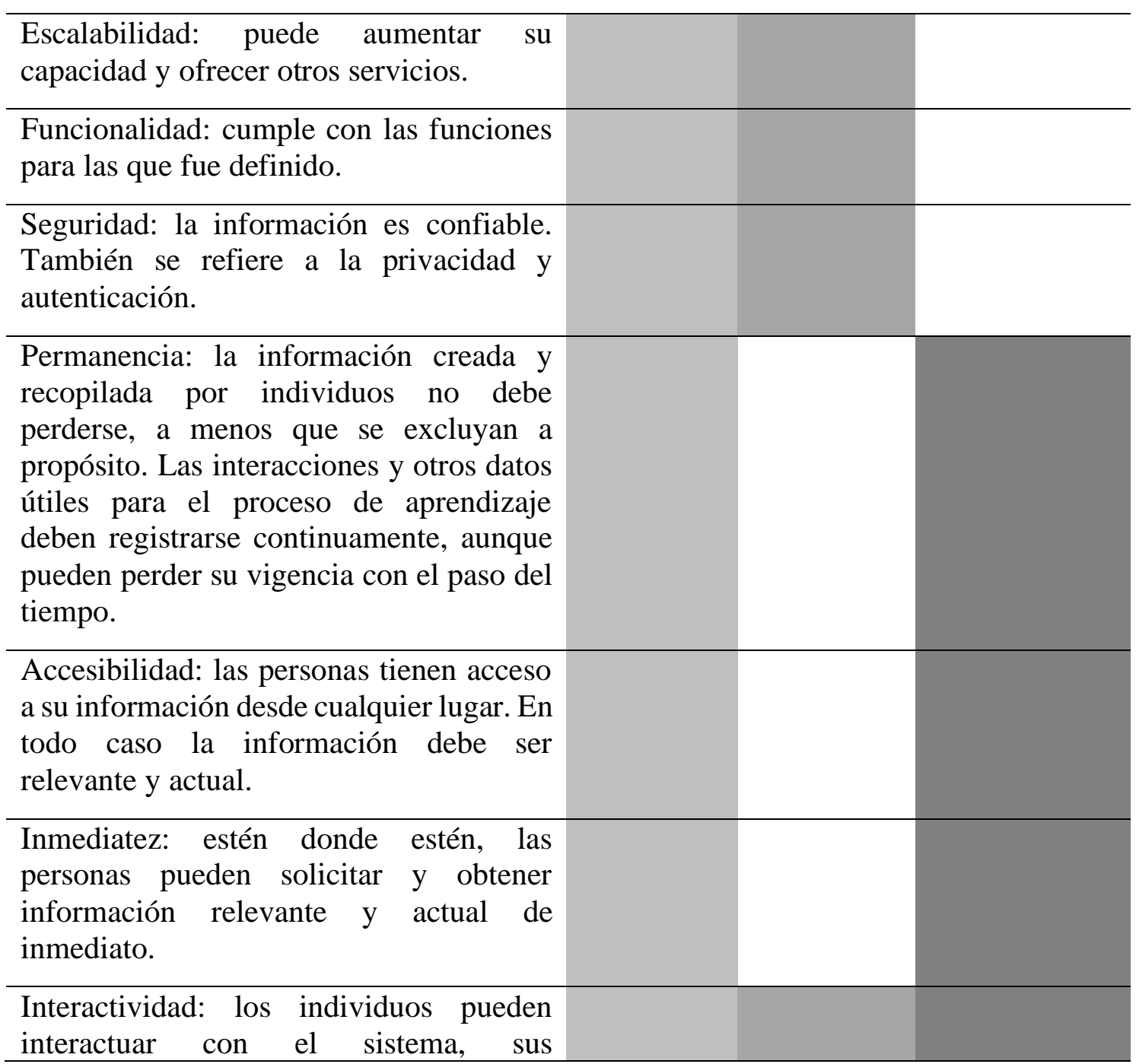

Análisis crítico del concepto "aprendizaje ubicuo" a través de la Cartografía Conceptual. Belén Velázquez Gatica y Rocío Edith López Martínez. 


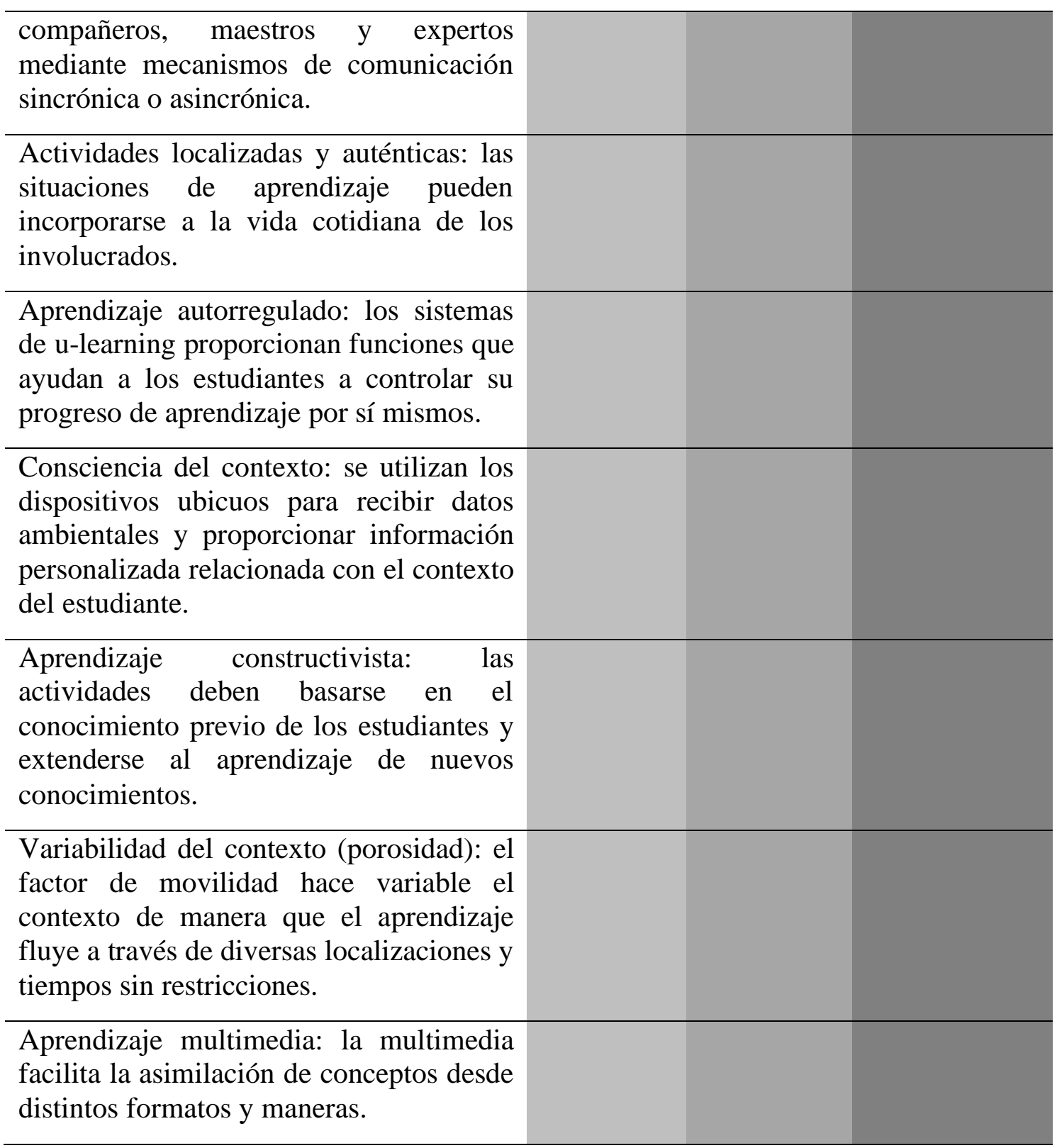

Fuente: elaboración propia a partir de datos recopilados en Brazuelo \& Gallego (2014), Cantú-Cervantes et al., (2019), de Sousa et al., (2014), Ferreira \& Castilho (2018), García-Sánchez \& Santos-Espino (2017), Heredia (2016), Huang et al., (2011), Manolakis (2011), Marinagi et al., (2013), Mojarro-Aliaño et al., (2015), Moreno et al., (2016), Moreno-López et al., (2017), Páramo et al., (2015), Rodrigo y de Castro (2013), Wang \& Wu (2011).

A partir del análisis de las características de los entornos de aprendizaje ubicuo se puede concluir que la ubicuidad en el aprendizaje no es una propiedad de algo o de alguien, sino una posibilidad que se configura a partir de la conjunción de distintos elementos que hacen el concepto potente en una situación ideal de aprendizaje. Dado que los elementos previamente descritos junto con su respectiva caracterización configuran un escenario ideal de aprendizaje ubicuo pueden proporcionar las bases para la evaluación de la calidad de entornos de $u$-learning, priorizando siempre los componentes que se vinculan con el eje de pedagogía y flujos de aprendizaje y permeados en todo momento por un objetivo de aprendizaje. 


\subsection{Diferenciación: ¿De cuáles otros enfoques cercanos se diferencia el aprendizaje ubicuo?}

El aprendizaje ubicuo en su noción tecnocéntrica se diferencia de otros tipos de aprendizaje mediados por tecnologías que son similares, como el m-learning y los entornos personales de aprendizaje (ver Tabla 3). Asimismo, desde su perspectiva como enfoque social regulador de prácticas de aprendizaje, se asemeja a conceptos como el aprendizaje rizomático, el aprendizaje invisible y la educación expandida (ver Tabla 4). Dentro del primer grupo, el aprendizaje ubicuo comparte algunos aspectos en común con el m-learning y los Entornos Personales de Aprendizaje:

1. Enfatizan el aprendizaje en contextos reales,

2. buscan la personalización del aprendizaje puesto que están centrados en la perspectiva del individuo que aprende,

3. deslocalizan y amplían los lugares y momentos de aprendizaje que se limitaban al espacio y horario escolar

Tabla 3

Diferencias entre el u-learning, m-learning y los entornos personales de aprendizaje

\begin{tabular}{|c|c|c|c|}
\hline Aspecto & u-learning & m-learning & $\begin{array}{l}\text { Entorno personal de } \\
\text { aprendizaje }\end{array}$ \\
\hline $\begin{array}{l}\text { ¿Qué?: } \text { Medios } \\
\text { digitales en los } \\
\text { que se apoya } \\
\text { para } r \\
\text { aprendizaje }\end{array}$ & $\begin{array}{lr}\text { Aprendizaje } & \text { en } \\
\text { cualquier dispositivo } \\
\text { digital móvil o fijo. } \\
\text { Énfasis en la } \\
\text { integración para el } \\
\text { aprendizaje }\end{array}$ & $\begin{array}{l}\text { Aprendizaje en } \\
\text { diferentes } \\
\text { dispositivos } \\
\text { móviles. Énfasis } \\
\text { en la movilidad del } \\
\text { aprendizaje }\end{array}$ & $\begin{array}{l}\text { Aprendizaje en un } \\
\text { espacio educativo } \\
\text { previamente } \\
\text { construido por el } \\
\text { aprendiz. Énfasis en } \\
\text { la estabilidad del } \\
\text { aprendizaje }\end{array}$ \\
\hline $\begin{array}{ll}\text { ¿Quién?: } & \\
\text { Cualidad } & \\
\text { principal del } \\
\text { sujeto } \\
\text { aprende } & \\
\end{array}$ & $\begin{array}{l}\text { Aprendiz en cualquier } \\
\text { lugar y en cualquier } \\
\text { momento }\end{array}$ & $\begin{array}{l}\text { Aprendiz } \\
\text { dispositivos } \\
\text { móviles }\end{array}$ & $\begin{array}{lr}\text { Aprendiz con } \\
\text { consciencia de su } \\
\text { entorno educativo }\end{array}$ \\
\hline $\begin{array}{l}\text { ¿Cómo?: } \\
\text { Forma en la que } \\
\text { sucede el } \\
\text { aprendizaje }\end{array}$ & $\begin{array}{l}\text { Flujo natural de } \\
\text { aprendizaje que se } \\
\text { modifica } \\
\text { constantemente por } \\
\text { algún motivo. } \\
\text { Aprendizaje dinámico, } \\
\text { flexible }\end{array}$ & $\begin{array}{l}\text { Flujo continuo de } \\
\text { aprendizaje } \\
\text { través } \\
\text { dispositivos } \\
\text { móviles }\end{array}$ & $\begin{array}{l}\text { Flujo habitual de } \\
\text { aprendizaje }\end{array}$ \\
\hline $\begin{array}{l}\text { ¿Cuándo?: } \\
\text { Momentos } \\
\text { aprendizaje }\end{array}$ & \begin{tabular}{lr}
\multicolumn{2}{l}{ En cualquier momento } \\
que se necesite, \\
incluyendo & nuevos \\
momentos para el \\
aprendizaje
\end{tabular} & $\begin{array}{l}\text { Aprendizaje } \\
\text { movimiento }\end{array}$ & $\begin{array}{l}\text { Momentos habituales } \\
\text { de aprendizaje }\end{array}$ \\
\hline $\begin{array}{l}\text { ¿Dónde?: } \\
\text { Espacios } \\
\text { aprendizaje }\end{array}$ & $\begin{array}{l}\text { En cualquier lugar que } \\
\text { se requiera, incluyendo } \\
\text { el uso de nuevos }\end{array}$ & $\begin{array}{l}\text { En los dispositivos } \\
\text { móviles, } \\
\text { agregando nuevos }\end{array}$ & $\begin{array}{l}\text { Espacio educativo } \\
\text { habitual construido } \\
\text { por el aprendiz }\end{array}$ \\
\hline
\end{tabular}

Análisis crítico del concepto "aprendizaje ubicuo" a través de la Cartografía Conceptual. Belén Velázquez Gatica y Rocío Edith López Martínez. 


$$
\begin{array}{lll}
\begin{array}{l}
\text { espacios para } \\
\text { aprendizaje }
\end{array} & \text { el } \begin{array}{l}
\text { dispositivos } \\
\text { ofertados }
\end{array}
\end{array}
$$

Fuente: elaboración propia a partir de datos recopilados en Báez \& Clunie (2019), Bernacki, Greene, et al., (2020), Burden et al., (2019), García (2017), Huang et al., (2008), Humanante-Ramos et al., (2017), Hwang et al., (2009), Karimi (2016), Lucke \& Rensing (2013), Marín (2016), Moreno et al., (2016), Park (2011), Pimmer et al., (2016), Quintero et al., (2015).

En cuanto a los enfoques sociales reguladores de prácticas educativas, hay una serie de semejanzas entre el aprendizaje ubicuo y el aprendizaje rizomático, la educación expandida y el aprendizaje invisible. Entre ellas se encuentran las siguientes (CastañoGarrido et al., 2018; Uribe, 2017):

1. Defienden una concepción ampliada frente a la educación y la pedagogía;

2. diferencian entre escolarización y educación;

3. aceptan que los lugares y los tiempos para el aprendizaje se están ensanchando y difuminando;

4. rescatan que las reflexiones educativas ya no se circunscriben exclusivamente al ámbito formal.

No obstante, presentan algunas diferencias en cuanto a ciertos aspectos de la educación (ver Tabla 4).

Tabla 4

\begin{tabular}{|c|c|c|c|c|}
\hline Aspecto & $\begin{array}{l}\text { Aprendizaje } \\
\text { ubicuo }\end{array}$ & $\begin{array}{l}\text { Aprendizaje } \\
\text { rizomático }\end{array}$ & $\begin{array}{l}\text { Aprendizaje } \\
\text { invisible }\end{array}$ & $\begin{array}{l}\text { Educación } \\
\text { expandida }\end{array}$ \\
\hline $\begin{array}{l}\text { Origen de } \\
\text { la } \\
\text { propuesta }\end{array}$ & $\begin{array}{l}\text { Crítica hacia la } \\
\text { subutilización } \\
\text { de la } \\
\text { tecnología, los } \\
\text { espacios y el } \\
\text { tiempo para } \\
\text { realizar } \\
\text { prácticas de } \\
\text { aprendizaje }\end{array}$ & $\begin{array}{lr}\text { Crítica a las } \\
\text { estructuras } & \text { de } \\
\text { organización } & \text { que } \\
\text { restringen } & \text { el } \\
\text { aprendizaje } & \end{array}$ & $\begin{array}{l}\text { Crítica hacia el } \\
\text { aprendizaje } \\
\text { permanente } \\
\text { como algo que } \\
\text { debe transitar de } \\
\text { lo discursivo a } \\
\text { lo real en todos } \\
\text { los niveles }\end{array}$ & $\begin{array}{l}\text { Crítica hacia la } \\
\text { escuela y su } \\
\text { discurso } \\
\text { hegemónico }\end{array}$ \\
\hline $\begin{array}{l}\text { Relación } \\
\text { entre } \\
\text { aprendizaje } \\
\text { formal-no } \\
\text { formal }\end{array}$ & $\begin{array}{l}\text { Deslocalizado. } \\
\text { El aprendizaje } \\
\text { se da en } \\
\text { cualquier lugar } \\
\text { y en cualquier } \\
\text { momento }\end{array}$ & $\begin{array}{l}\text { Deslocalizado. } \\
\text { Es difícil } \\
\text { establecer sus } \\
\text { fronteras debido } \\
\text { a que es caótico, } \\
\text { expansivo y } \\
\text { multidireccional }\end{array}$ & $\begin{array}{l}\text { Deslocalizado. } \\
\text { El aprendizaje } \\
\text { se adapta desde } \\
\text { la especificidad } \\
\text { y diversidad de } \\
\text { cada contexto }\end{array}$ & $\begin{array}{l}\text { Énfasis en el } \\
\text { aprendizaje } \\
\text { informal. La } \\
\text { educación } \\
\text { sucede } \\
\text { esencialmente } \\
\text { fuera de las } \\
\text { instituciones } \\
\text { educativas }\end{array}$ \\
\hline $\begin{array}{l}\text { Cualidad } \\
\text { del sujeto } \\
\text { que } \\
\text { aprende }\end{array}$ & $\begin{array}{ll}\text { Aprendiz } & \text { que } \\
\text { sigue } & \text { un } \\
\text { proceso } & \text { de } \\
\text { aprendizaje } & \text { sin } \\
\text { restricciones } & \end{array}$ & $\begin{array}{l}\text { Aprendiz con } \\
\text { objetivos de } \\
\text { aprendizaje } \\
\text { personalizados, } \\
\text { pero no regulares }\end{array}$ & $\begin{array}{l}\text { Aprendiz dentro } \\
\text { de un proceso } \\
\text { continuo } \\
\text { informal que } \\
\text { aprende a través } \\
\text { de las }\end{array}$ & $\begin{array}{l}\text { Aprendiz que } \\
\text { simplemente } \\
\text { desea saber } \\
\text { algo }\end{array}$ \\
\hline
\end{tabular}

Diferencias entre el aprendizaje ubicuo, rizomático, invisible y la educación expandida

Análisis crítico del concepto "aprendizaje ubicuo" a través de la Cartografía Conceptual. Belén Velázquez Gatica y Rocío Edith López Martínez. 


\begin{tabular}{|c|c|c|c|c|}
\hline & & & $\begin{array}{l}\text { interacciones } \\
\text { cotidianas }\end{array}$ & \\
\hline $\begin{array}{l}\text { Objetivo de } \\
\text { la } \\
\text { propuesta }\end{array}$ & $\begin{array}{l}\text { Promover el } \\
\text { dominio del } \\
\text { aprendizaje } \\
\text { sobre el espacio } \\
\text { y el tiempo }\end{array}$ & $\begin{array}{l}\text { Crear un contexto } \\
\text { dentro del cual el } \\
\text { plan de estudios y } \\
\text { el conocimiento } \\
\text { se construyan por } \\
\text { los miembros de } \\
\text { una } \\
\text { comunidad de } \\
\text { aprendizaje y que } \\
\text { puede } \\
\text { reformado der } \\
\text { manera dinámica. } \\
\text { Utilizar una } \\
\text { diversidad } \\
\text { medios de } \\
\text { homogéneos no }\end{array}$ & $\begin{array}{l}\text { Integrar } \\
\text { diversos } \\
\text { enfoques en } \\
\text { relación con un } \\
\text { nuevo } \\
\text { paradigma de } \\
\text { aprendizaje y } \\
\text { desarrollo del } \\
\text { capital humano, } \\
\text { relevante en el } \\
\text { marco del siglo } \\
\text { XXI }\end{array}$ & $\begin{array}{l}\text { Comprender } \\
\text { aquellos } \\
\text { aprendizajes } \\
\text { que son } \\
\text { invisibles para } \\
\text { la formalidad } \\
\text { escolar. } \\
\text { Democratizar } \\
\text { el aprendizaje }\end{array}$ \\
\hline
\end{tabular}

Fuente: elaboración propia a partir de datos recopilados en Cabero \& Llorente (2015), Cobo \& Moravec (2011), Uribe-Zapata (2018).

Entre las principales diferencias del aprendizaje ubicuo con los demás conceptos y enfoques se encuentra la generalización del aprendizaje continuo y la preponderancia del sujeto que aprende respecto a la variedad de tecnologías, modalidades y espacios formales e informales, puesto que aboga por una integración más que por el dominio o la preferencia de un medio, espacio o tiempo. Sin embargo, esta falta de reconocimiento de las situaciones específicas de aprendizaje se está modificando en la propuesta del aprendizaje ubicuo, a diferencia de otros enfoques como el aprendizaje rizomático y la educación expansiva. Dicha modificación se está realizando a través de la integración de diferentes elementos que configuran un contexto de aprendizaje basado en preferencias.

\subsection{Clasificación: ¿En qué subclases o tipos se clasifica el aprendizaje ubicuo?}

En la literatura especializada sobre el aprendizaje ubicuo no se encontraron criterios de clasificación del concepto. No obstante, existe un tipo de aprendizaje ubicuo que se ha utilizado para diferenciar aquellos entornos y sistemas que se construyen, con base en el $u$-learning como un proceso personalizado, que depende del contexto y que en ocasiones combina elementos del mundo físico y del virtual para resolver problemas de aprendizaje reales (G. J. Hwang et al., 2011; Kim et al., 2012; Souabni et al., 2017), de otros que se enfocan en la generalización del aprendizaje y su accesibilidad.

Este tipo de aprendizaje ubicuo se denomina u-learning consciente del contexto. Su esencia consiste en buscar constantemente la personalización del aprendizaje de los usuarios, a través de diferentes estrategias y técnicas que les proporcionan recomendaciones o sugerencias útiles para tomar decisiones. Para ello, aprovecha la información contextual o datos contextuales que los estudiantes proveen a través de diferentes medios, en entornos distintos (Aguilar et al., 2017; C.-C. Chen \& Huang, 2012; Chu et al., 2010; Raza \& Ding, 2019). 
En el aprendizaje ubicuo consciente del contexto también existen variaciones respecto a lo que se entiende por contexto. Algunas definiciones están basadas en una visión individualizada como sinónimo de personalización del aprendizaje. En estos casos, los datos como la ubicación o el tiempo son suficientes para emitir recomendaciones de aprendizaje. Otras definiciones de lo que implica el contexto utilizan información como la infraestructura del sistema donde se encuentra el estudiante, su actividad educativa, sus interacciones con otros aprendices, así como otros datos que proporcionan los medios para la participación del alumno, aun cuando este no sea consciente del proceso (Marinagi et al., 2013) o bien, el u-learning consciente del contexto puede servir para dar soporte y guiar actividades de aprendizaje cooperativas y auténticas en tiempo real ya prediseñadas, incluyendo no solo el componente tecnológico sino el pedagógico (Crompton, 2015). Es decir, se asume que el proceso de aprendizaje es una actividad colectiva.

En términos generales, en el marco del u-learning, el contexto puede ser cualquier información relevante que pueda utilizarse para caracterizar una situación de aprendizaje y proporcionar servicios y recursos educativos relevantes al usuario (Pradeep \& Krishnamoorthy, 2019; Souabni et al., 2017). Las preguntas centrales que intenta responder son ¿quién aprende?, ¿qué?, ¿dónde?, ¿cuándo?, ¿cómo?, ¿con quién? y ¿por qué? Esta información se genera constantemente a partir de las interacciones que el individuo que aprende tiene con cualquier entidad, ya sea física (como una persona, un dispositivo u objeto), digital (un mensaje, un servicio informático) o un concepto (ubicación, hora, etc.). Es decir, el contexto surge de la actividad y por lo tanto puede cambiar en cualquier momento por algún motivo.

\subsection{Ejemplificación: ¿Cuál sería un ejemplo relevante y pertinente de aplicación del aprendizaje ubicuo?}

Los ejemplos sobre la aplicación del concepto aprendizaje ubicuo pueden ser diversos y dependen del enfoque que se utiliza para la construcción del entorno y la experiencia $u$ learning. Algunos enfoques están más centrados en construir o mejorar el sistema tecnológico que dará soporte al aprendizaje ubicuo para garantizar el flujo de aprendizaje, sin considerar la pedagogía, el diseño instruccional, el objetivo de aprendizaje o la situación específica de aprendizaje. Los trabajos de Yang et al., (2013) y de Osman (2018) nos proporcionan ejemplos de este tipo de aplicaciones del concepto u-learning desde una perspectiva tecnocéntrica.

Otros trabajos se configuran en el marco de situaciones específicas de aprendizaje, en cuyo caso se integran las necesidades de aprendizaje de los estudiantes, el sistema, herramientas o estrategias $u$-learning más idóneas y se determina el campo de aplicación o la asignatura de aprendizaje y la pedagogía o el diseño instruccional que guiará la experiencia. Ejemplos de estas experiencias educativas pueden encontrarse en Huang \& Lin (2017), Hwang (2014), Kong (2019), Ranga (2018), Valenzuela-Valdés (2016), Wen \& Zhang (2014).

Finalmente, un enfoque de trabajos sobre el u-learning considera los aspectos previamente mencionados y aplica el concepto específico de aprendizaje ubicuo consciente del contexto, para diseñar actividades educativas caracterizadas por combinar elementos físicos y digitales para resolver problemas auténticos, muchos de ellos en tiempo real. Algunos ejemplos de estos trabajos pueden observarse en Chen \& Lin (2014), Crompton (2015), Hwang et al., (2011) y Hwang et al., (2020).

Análisis crítico del concepto "aprendizaje ubicuo" a través de la Cartografía Conceptual. Belén Velázquez Gatica y Rocío Edith López Martínez. 
El contraste entre los ejemplos de aplicaciones del concepto aprendizaje ubicuo se debe principalmente a la variedad de enfoques del u-learning, aunado al hecho de que el aprendizaje ubicuo carece de una metodología específica, siendo más una teoría de tipo descriptiva sobre los flujos de aprendizaje actuales que se posibilitan gracias a la convergencia tecnológica, que una teoría prescriptiva o instruccional.

\subsection{Vinculación: ¿Cómo se relaciona el aprendizaje ubicuo con determinadas teorías, procesos sociales-culturales $y$ referentes epistemológicos que están por fuera de la categorización?}

La publicidad ubicua se define como el uso de tecnologías ubicuas con fines publicitarios y se ha utilizado para llenar los vacíos que aún enfrenta la publicidad tradicional (Martinez-Pabon et al., 2014). En la publicidad también se emplean los medios digitales más novedosos para el mercadeo general, tal es el caso de la señalización digital. Por ejemplo, el uso de pantallas en ciertos lugares. No obstante, este tipo de pantallas se ubican cerca de los sitios de decisión de compra. Por su parte, la publicidad ubicua preferentemente se sustenta en los dispositivos móviles debido a que promueven un esquema de interacción simple (Martinez-Pabon et al., 2014).

En este sentido, la publicidad general digital es vertical, indirecta y pasiva, a diferencia de la publicidad ubicua que promueve una interacción horizontal con los usuarios en un nivel más participativo, íntimo y personalizado, que permite entregar información relevante en el momento y el contexto adecuado (Karchoud et al., 2017; Martinez-Pabon et al., 2014). La personalización es un concepto clave en la publicidad ubicua y se respalda del ajuste de las preferencias y el grado de involucramiento de los usuarios, de tal forma que, el cliente es un individuo activo en la concepción y despliegue de los productos del mercado.

En términos generales, la publicidad ubicua tiene tres objetivos principales: sugerir la compra de productos, advertir sobre artículos que deben evitarse, e identificar oportunidades mediante el análisis de perfiles y senderos para aumentar la probabilidad de que los clientes encuentren productos que satisfagan sus necesidades (Victória et al., 2016).

En resumen, la publicidad se complementa de las estrategias utilizadas en la publicidad tradicional y ubicua. La primera es vertical, observa al cliente como un personaje pasivo, se dirige a un público más general y su intención es inyectar la intención de compra de un producto o servicio tal y como está diseñado. La segunda es horizontal, no solo permite la participación del cliente, sino que se vale de sus preferencias e involucramiento para la personalización de recomendaciones. Su intención es dar la mejor alternativa de compra de entre un conjunto de productos o servicios. Asimismo, provee información para modificar o mejorar productos o servicios de acuerdo con información relevante proporcionada por los clientes.

La integración de la publicidad general y la publicidad ubicua es análoga al aprendizaje ubicuo y el aprendizaje ubicuo consciente del contexto, debido a que persiguen objetivos semejantes, pero desde sus propios mecanismos. Asimismo, los procesos educativos en ambos tipos de aprendizaje están interrelacionados. En este sentido, una forma de complementar ambos procesos sería en dos direcciones. Desde el $u$-learning se inyectaría una necesidad de aprendizaje desde lo general, durante el proceso se recogen aspectos

Análisis crítico del concepto "aprendizaje ubicuo" a través de la Cartografía Conceptual. Belén Velázquez Gatica y Rocío Edith López Martínez. 
relevantes del mismo y estos pueden utilizarse para modificar la forma y el flujo de aprendizaje personal.

O bien, desde el u-learning consciente del contexto se partiría de un proceso de aprendizaje autoiniciado, en el proceso se recogen las preferencias y se puede utilizar para modificar la forma y el flujo de aprendizaje general. Es decir, se debe aprovechar el contenido de aprendizaje y sus flujos, que transitan de lo global a lo individual y viceversa, y entre escenarios, para integrar más que para excluir. De tal manera que, se puedan llenar los vacíos que se producen en ambos tipos de u-learning, tanto el general como el consciente del contexto, en un entorno de aprendizaje ubicuo mixto.

\section{Discusión}

El término aprendizaje ubicuo ha ganado relevancia en los últimos años, debido a la importancia que involucra el uso de la tecnología para aprender en cualquier lugar y en cualquier momento. Como concepto, el u-learning se originó en el marco de la computación ubicua, donde el interés estaba en la naturalización del uso de las computadoras en las actividades de las personas. Sin embargo, desde esta perspectiva el foco estaba en las tecnologías y en sus posibilidades, más que en los aspectos pedagógicos y sociales. Por esta razón, el aprendizaje ubicuo se ha criticado debido a su carácter tecnocéntrico.

No obstante, el concepto ganó madurez gracias a la incorporación de enfoques educativos como el aprendizaje continuo y el aprendizaje personalizado. Esto permitió que el $u$ learning evolucionara conceptualmente en términos educativos, y se hiciera potente para fines de aprendizaje, en lugar de mantener la perspectiva aplicacionista de la tecnología en el contexto educativo. A pesar de esta evolución, en la literatura especializada aún existen documentos basados en enfoques tecnocéntricos del aprendizaje ubicuo. Al respecto, Jung (2014) refirió que las investigaciones previas sobre aprendizaje ubicuo han sido limitadas, puesto que la mayoría se han centrado en la tecnología de $u$-learning en sí misma, en lugar de comprender el comportamiento de u-learning de los alumnos.

Otra evolución paralela del concepto involucró el abandono del u-learning como un aprendizaje generalizado y su transición hacia un aprendizaje en contexto, es decir, que depende de una situación educativa específica, así como de las preferencias de los estudiantes, su ubicación, sus interacciones, entre otros aspectos que actualmente configuran el aprendizaje ubicuo consciente del contexto.

Considerando el contexto, los entornos y sistemas de aprendizaje ubicuo actuales intentan construir un escenario educativo en donde se integren las posibilidades tecnológicas, los flujos de aprendizaje, aspectos pedagógicos y las características del individuo que aprende, debido a que, si se separan dichos elementos, el aprendizaje ubicuo reduce su potencial. Es decir, la tecnología puede ser ubicua, pero sin objetivos de aprendizaje reales, pierde su propósito educativo y no hay un verdadero $u$-learning.

Cabe destacar que en el aprendizaje ubicuo el uso de la tecnología es una condición necesaria, puesto que es innegable que la convergencia impulsó los procesos de distribución y difusión de la cultura y del conocimiento actuales (Ferreira \& Castilho, 2018). No obstante, no es una cualidad suficiente ni deseable para propósitos educativos. De hecho, algunos autores han señalado que la tecnología debe combinarse con métodos efectivos de construcción del conocimiento, puesto que sin las estrategias de aprendizaje

Análisis crítico del concepto "aprendizaje ubicuo" a través de la Cartografía Conceptual. Belén Velázquez Gatica y Rocío Edith López Martínez. 
o las herramientas mentales eficaces, el aprendizaje ubicuo puede ser decepcionante para los aprendices (G.-J. Hwang et al., 2020; G. J. Hwang et al., 2011).

De igual forma, dentro del mero aspecto tecnológico, los dispositivos móviles consiguen ser ubicuos gracias a los servicios de conexión a internet. Aunado a ello, la idea de la ubicuidad no es novedosa en términos pedagógicos, por el contrario, es anterior a la aparición de los dispositivos tecnológicos, con las constantes innovaciones educativas, comenzando con el concepto de "educación para la vida" de Dewey (Marinagi et al., 2013).

Es decir, la ubicuidad en el aprendizaje no es un atributo de algo o de alguien, contrario a lo que se refiere en la definición común de ubicuidad, la cual se describe como un adjetivo "Que está a un mismo tiempo en todas partes" (Real Academia Española, 2014). En contraste, la ubicuidad en el aprendizaje es una propiedad que se vuelve posible a partir de la vinculación de las características de distintos elementos.

En este texto, se han analizado un conjunto de propiedades que definen un entorno ideal de aprendizaje u-learning y que por lo tanto pueden servir de guía para la construcción de entornos de aprendizaje ubicuos, así como para la evaluación de su calidad. Cabe destacar que en dicha valoración, no solo será pertinente evaluar la inclusión de las características que se observan en la Tabla 2. En un nivel más elevado, también será necesario estimar la coherencia entre estos elementos que interactúan entre sí, y su relevancia y contribución con relación al objetivo final de aprendizaje.

En cuanto al significado actual del aprendizaje ubicuo se puede describir como un enfoque educativo apoyado en la ubicuidad de la tecnología, que busca, a través de un discurso, la interiorización del dominio del aprendizaje sobre los tiempos y espacios para mantener un flujo continuo de aprendizaje. Esto se ilustra en la Figura 1.

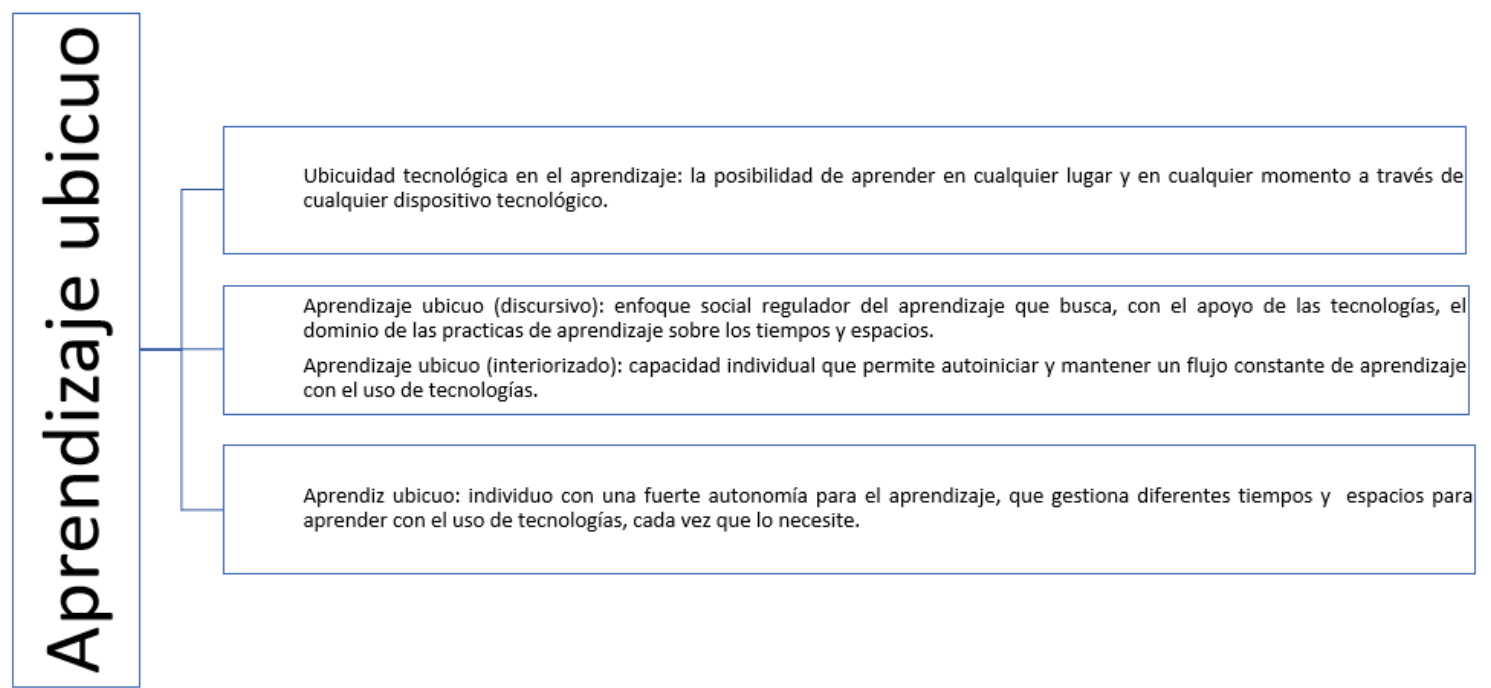

Figura 1. Configuración del concepto actualizado del aprendizaje ubicuo y sus aspectos distintivos

Fuente: Elaboración propia.

Sin embargo, el aprendizaje ubicuo alude a un enfoque educativo ideal y la construcción de tales condiciones, por ejemplo, en un entorno o sistema de aprendizaje ubicuo. No obstante, es necesario incorporar otros conceptos que apoyen la perspectiva del $u$ learning, a la hora de establecer prácticas educativas concretas, que permitan una

Análisis crítico del concepto "aprendizaje ubicuo" a través de la Cartografía Conceptual. Belén Velázquez Gatica y Rocío Edith López Martínez. 
aplicación más real del concepto. Estos conceptos apuntan en su mayoría a la forma en cómo se configuran las prácticas sociales educativas cotidianas, a partir de las posibilidades, pero también de las limitaciones del aprendizaje en contexto (ver Figura 2).

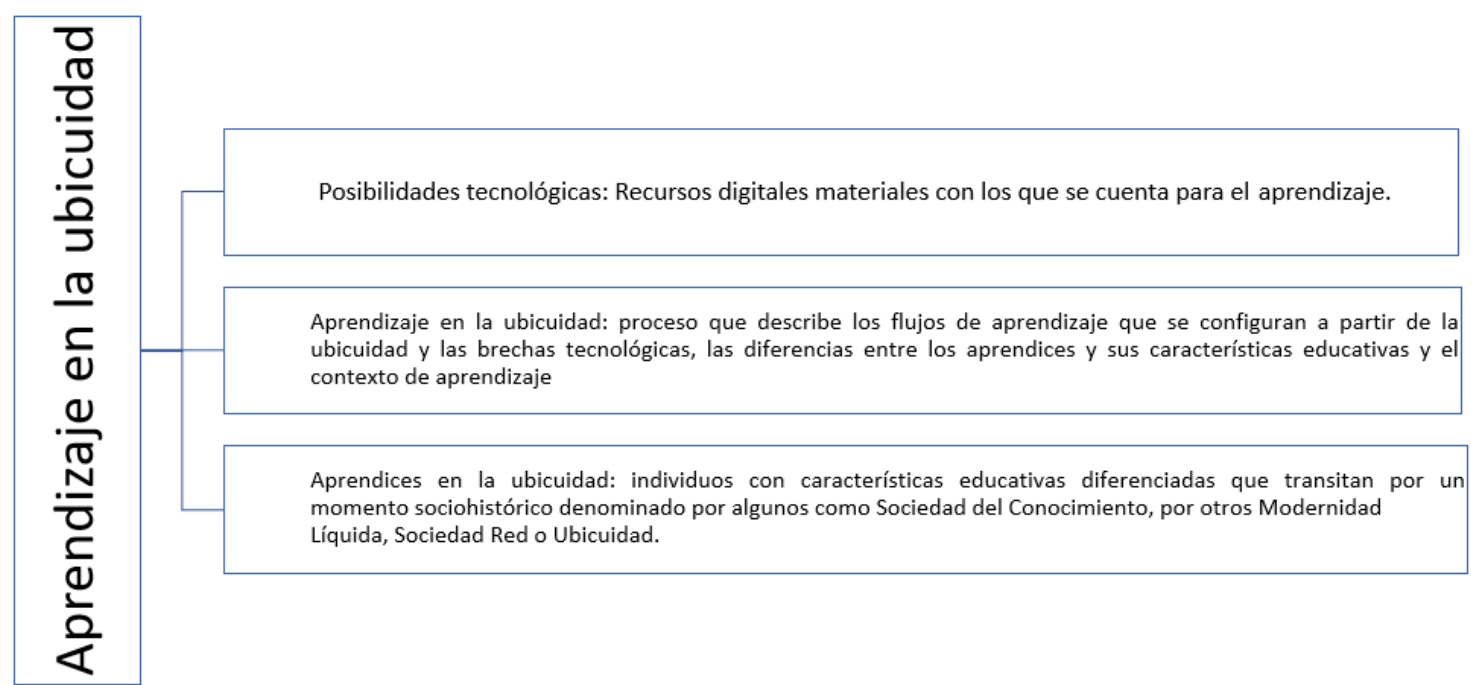

Figura 2. Propuesta del concepto aprendizaje en la ubicuidad y sus aspectos distintivos

Fuente: Elaboración propia.

La diferencia entre el aprendizaje ubicuo y el aprendizaje en la ubicuidad es que el primero describe una situación ideal de aprendizaje con tecnologías y gestión de espacios y tiempos, mientras que el segundo describe un proceso de aprendizaje y sus flujos cotidianos, como acontecen actualmente en el marco de diferentes elementos contextuales, tanto idóneos como limitantes. En este sentido, existe una necesidad de investigar y documentar sobre la forma en cómo se dan actualmente estos flujos y la manera en que este conocimiento puede ayudar a configurar escenarios de aprendizaje ubicuo, ya sean generales, conscientes del contexto o mixtos, para que el enfoque de aprendizaje ubicuo sea una realidad.

Dadas las limitaciones tecnológicas en algunos contextos educativos que configuran la brecha digital, la forma en la que se puede impulsar el $u$-learning pese a estas restricciones es a partir de fomentar la actividad continua del aprendiz, es decir, la formación del aprendizaje ubicuo interiorizado que permita reducir las brechas entre aprendices. Lo anterior se logrará a través de la inclusión de teorías pedagógicas que orienten las posibilidades del u-learning, puesto que este enfoque se trata más de una teoría del aprendizaje que de una teoría pedagógica o práctica.

La diferencia entre ambos tipos de teorías estriba en que las primeras se centran en la descripción de los aspectos del proceso de aprendizaje en donde el protagonista es el aprendiz, mientras que las segundas son de naturaleza prescriptiva y se enfocan en los métodos de instrucción que hay que utilizar para conseguir objetivos educativos y por lo tanto en el rol del diseñador y del instructor (Sobrino, 2011; Zapata-Ros, 2015b). No obstante, el aprendizaje ubicuo se trata de una teoría que intenta explicar el aprendizaje y que a su vez tiene rasgos normativos sin un método de instrucción específico, es decir, es prescriptivo de manera general, puesto que en su discurso pretende fomentar en los

Análisis crítico del concepto "aprendizaje ubicuo" a través de la Cartografía Conceptual. Belén Velázquez Gatica y Rocío Edith López Martínez. 
aprendices la capacidad de amplificar las oportunidades de crear actividades y entornos para llevar a cabo múltiples aprendizajes, a través de las potencialidades tecnológicas (Collazos et al., 2016).

En este sentido, el aprendizaje ubicuo carece de una metodología, por ello, algunos autores afirman que se le ha dado mucha importancia a las necesidades del aprendiz (Gilman et al., 2015) y a los atributos de la tecnología (Jung, 2014), mientras que se ha prescindido del aspecto pedagógico que oriente una experiencia educativa integral. Por lo tanto, el aprendizaje ubicuo debe complementarse con una teoría pedagógica que también sea acorde a las necesidades educativas actuales. Una de estas opciones la constituye la socioformación, debido a que se trata de un enfoque que busca que los estudiantes desarrollen su talento y se realicen plenamente a través de la resolución de problemas de la sociedad real, desde el contexto local con una visión global, con base en sus propias vivencias y seleccionando área concretas de actuación, así como gestionando el conocimiento en diferentes fuentes con sentido crítico y trabajo colaborativo (Tobón et al., 2015). Algo que es acorde con el aprendizaje ubicuo consciente del contexto, el cual combina elementos físicos y digitales para resolver problemas auténticos en tiempo real con alto grado de significancia.

Por lo tanto, un enfoque general de $u$-learning que incluye la construcción de experiencias educativas y entornos de aprendizaje ubicuo consciente del contexto y centrado en el aprendizaje debe partir y guiarse en todo momento de la formación de los aprendices como eje vertebrador de la articulación de objetivos y flujos de aprendizaje, pedagogías, métodos y por supuesto, contando con todas las ventajas que ofrecen hoy en día las tecnologías (Coto et al., 2016). En consecuencia, las tecnologías no deben marcar los objetivos y las trayectorias de aprendizaje, puesto que es más relevante el aprendizaje que se puede lograr a través de los dispositivos móviles como medio para acceder a personas, espacios en internet o la nube, recursos y contenidos que los dispositivos en sí (ZapataRos, 2015a), que en última instancia suelen ser secundarios, dada la diversidad de alternativas que se pueden utilizar.

En este caso, el aprendizaje ubicuo interiorizado se observa más como la capacidad de iniciar o mantener ciclos de aprendizaje que obedecen a objetivos de formación globales, que como un aprendizaje continuo con objetivos aislados apoyado por tecnologías. Debido a que las trayectorias y los flujos de aprendizaje actuales están constituidos por un conjunto de proyectos y situaciones de aprendizaje con tecnologías que se imbrican y que pueden ser autoiniciadas o emprendidas por otros individuos en el marco de las instituciones. Se caracterizan por tener un inicio y un fin, además, responden a intereses y objetivos cambiantes o estables que se suman, más que constituir un aprendizaje disperso y en cualquier lugar y en cualquier momento a través de diferentes dispositivos digitales, sin determinar la relevancia educativa para los propósitos del individuo que aprende (Ver Figura 3). En este sentido, todos los elementos que se encuentran en los ecosistemas de aprendizaje de un individuo, y no solo la tecnología, le brindan las posibilidades para que construya su propia trayectoria de aprendizaje a partir de sus necesidades o intereses, lo que se vuelve casi un hecho es que un aprendiz tendrá actividades de aprendizaje en casi todos sus periodos vitales (Islas \& Carranza, 2017).

Análisis crítico del concepto "aprendizaje ubicuo" a través de la Cartografía Conceptual. Belén Velázquez Gatica y Rocío Edith López Martínez. 


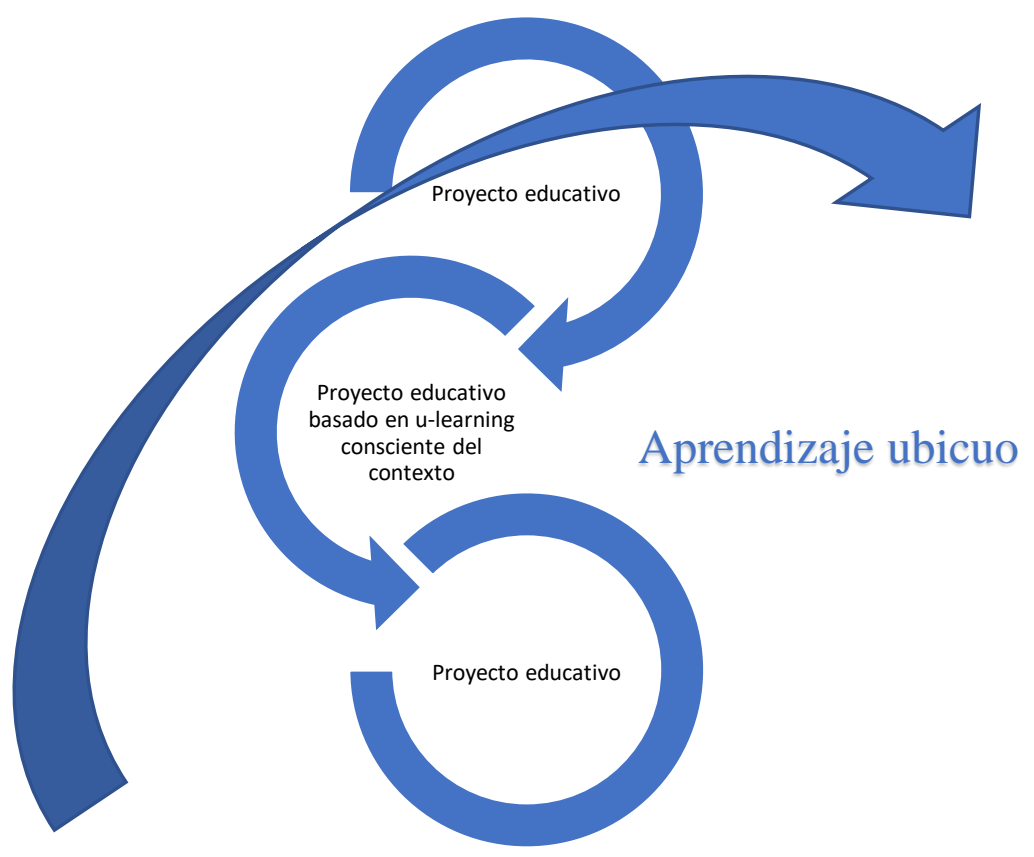

Figura 3. Enfoque general de aprendizaje ubicuo centrado en el aprendizaje.

Fuente: Elaboración propia

En este escenario los proyectos de aprendizaje suelen ser formales y no formales, de corto y mediano plazo, y brindan experiencias educativas planeadas, mientras que las situaciones de aprendizaje son más rápidas, no planeadas e imprevistas. Sin embargo, en las dos circunstancias un individuo puede realizar acciones relevantes y significativas para su proyecto educativo global.

El aprendizaje ubicuo consciente del contexto puede brindar las condiciones idóneas para configurar proyectos educativos que consigan resultados significativos, siempre que la consciencia del contexto incluya la construcción de experiencias educativas que conjuguen tecnología, estrategias y pedagogías culturalmente relevantes y de acuerdo con el tipo de proyecto educativo del que se trate. Un proyecto educativo puede ser una clase, un curso, un taller, un congreso, o un programa completo.

En resumen, para hacer efectivo el u-learning no es suficiente un enfoque tecnológico potente, sino que se debe acompañar de una teoría y un método en particular que también sea acorde con las exigencias de la educación actual y socialmente relevante para los aprendices.

Presentación del manuscrito: 03 de junio de 2020

Fecha de aprobación: 10 de octubre de 2020

Fecha de publicación: 30 de abril de 2021

Velázquez, B. \& López, R.E. (2021). Análisis crítico del concepto "aprendizaje ubicuo" a través de la Cartografía Conceptual. RED. Revista Educación a Distancia, 21(66). http://dx.doi.org/10.6018/red.430841

Análisis crítico del concepto "aprendizaje ubicuo" a través de la Cartografía Conceptual. Belén Velázquez Gatica y Rocío Edith López Martínez. 


\section{Financiación}

Este trabajo no ha recibido ninguna subvención específica de los organismos de financiación en los sectores públicos, comerciales o sin fines de lucro.

\section{Referencias}

Abascal, J., \& Moriyón, R. (2002). Tendencias en Interacción Persona Computador. Inteligencia Artificial. Revista Iberoamericana de Inteligencia Artificial, 6(16), 924.

Aguilar, J., Mendonça, M., Jerez, M., \& Sánchez, M. (2017). Emergencia ontológica basada en análisis de contexto, como servicio para ambientes inteligentes. Dyna, 84(200), 28-37. https://doi.org/10.15446/dyna.v84n200.59062

Artopoulos, A., \& Kozak, D. (2011). Tsunami 1:1: estilos de adopción de tecnología en la educación latinoamericana. Revista Iberoamericana de Ciencia, Tecnología y Sociedad-CTS, 6(18).

Báez, C. I., \& Clunie, C. E. (2019). Una mirada a la Educación Ubicua. RIED. Revista Iberoamericana de Educación a Distancia, 22(1), 325-344. https://doi.org/10.5944/ried.22.1.22422

Bernacki, M. L., Crompton, H., \& Greene, J. A. (2020). Towards convergence of mobile and psychological theories of learning. Contemporary Educational Psychology, 60, 1-7. https://doi.org/10.1016/J.CEDPSYCH.2019.101828

Bernacki, M. L., Greene, J. A., \& Crompton, H. (2020). Mobile technology, learning, and achievement: Advances in understanding and measuring the role of mobile technology in education. Contemporary Educational Psychology, 60, 1-8. https://doi.org/10.1016/j.cedpsych.2019.101827

Brazuelo, F., \& Gallego, D. J. (2014). Estado del Mobile Learning en España. Educar em Revista, Edición especial 4, 99-128. https://doi.org/10.1590/0104-4060.38646

Burden, K., Kearney, M., Schuck, S., \& Hall, T. (2019). Investigating the use of innovative mobile pedagogies for school-aged students: A systematic literature review. Computers and Education, 138, 83-100. https://doi.org/10.1016/j.compedu.2019.04.008

Cabero, J., \& Llorente, M. del C. (2015). Tecnologías de la Información y la Comunicación (TIC): escenarios formativos y teorías del aprendizaje. Revista Lasallista de Investigación, 12(2), 186-193.

Cantú-Cervantes, D., Amaya-Amaya, A., \& Baca-Pumarejo, J. R. (2019). Modelo para el reforzamiento del aprendizaje con dispositivos móviles. CienciaUAT, 13(2), 56-70. https://doi.org/10.29059/cienciauat.v13i2.1161

Cárdenas-Robledo, L. A., \& Peña-Ayala, A. (2018). Ubiquitous learning: A Systematic Review. Telematics and Informatics, 35(5), 1097-1132. https://doi.org/10.1016/j.tele.2018.01.009 
Casanovas, I., \& Tomassino, C. (2014). Capacitación profesional continua en la era de los dispositivos móviles. Revista Electrónica Gestión de las Personas y Tecnología, 7(21), 4-17.

Castaño-Garrido, C., Garay-Ruiz, U., \& Themistokleous, S. (2018). De la revolución del software a la del hardware en educación superior. RIED. Revista Iberoamericana de Educación a Distancia, 21(1). https://doi.org/10.5944/ried.21.1.18823

Chen, C.-C., \& Huang, T.-C. (2012). Learning in a u-Museum: Developing a contextaware ubiquitous learning environment. Computers and Education, 59(3), 873-883. https://doi.org/10.1016/j.compedu.2012.04.003

Chen, C.-C., \& Lin, P.-H. (2014). Development and evaluation of a context-aware ubiquitous learning environment for astronomy education. Interactive Learning Environments, 24(3), 644-661. https://doi.org/10.1080/10494820.2014.915417

Chen, G. D., Chang, C. K., \& Wang, C. Y. (2008). Ubiquitous learning website: Scaffold learners by mobile devices with information-aware techniques. Computers and Education, 50, 77-90. https://doi.org/10.1016/j.compedu.2006.03.004

Chu, H.-C., Hwang, G.-J., \& Tsai, C.-C. (2010). A knowledge engineering approach to developing mindtools for context-aware ubiquitous learning. Computers and Education, 54(1), 289-297. https://doi.org/10.1016/j.compedu.2009.08.023

Cobo, C., \& Moravec, J. W. (2011). Introducción al aprendizaje invisible: la (r)evolución fuera del aula. Reencuentro, 62, 66-81.

Collazos, C. A., Jurado, J. L., \& Merchán, L. (2016). Entornos ubicuos y colaborativos (U-CSCL) para ambientes de enseñanza-aprendizaje de competencias profesionales. Editorial Bonaventuriana.

Coto, M., Collazos, C. A., \& Mora, S. (2016). Modelo Colaborativo y Ubicuo para apoyar los procesos de enseñanza-aprendizaje a nivel Iberoamericano. RED-Revista de Educación a Distancia, 48(10). https://doi.org/10.6018/red/48/10

Crompton, H. (2015). Using Context-Aware Ubiquitous Learning to Support Students' Understanding of Geometry. Journal of Interactive Media in Education, 1(13), 111. https://doi.org/10.5334/jime.aq

De la Torre, J., Martin-Dorta, N., Saorín, J. L., Carbonell, C., \& Contero, M. (2013). Entorno de aprendizaje ubicuo con realidad aumentada y tabletas para estimular la comprensión del espacio tridimensional. Revista de Educación a Distancia, 12(37). http://www.um.es/ead/red/37

de Sousa, B., Gomes, A. S., \& Mendes, F. M. (2014). Youubi: Open software for ubiquitous learning. Computers in Human Behavior, 55, 1145-1164. https://doi.org/10.1016/j.chb.2014.09.064

El Guabassi, I., Bousalem, Z., Al Achhab, M., Jellouli, I., \& El Mohajir, B. E. (2018). Personalized adaptive content system for context-aware ubiquitous learning.

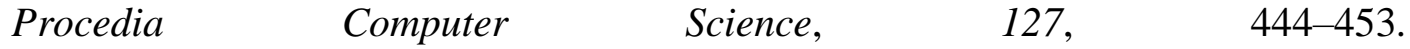
https://doi.org/10.1016/j.procs.2018.01.142

Fernández, E., \& Anguita, R. (2015). Aprendizajes invisibles en contextos de educación

Análisis crítico del concepto "aprendizaje ubicuo" a través de la Cartografía Conceptual. Belén 
expandida. Retos y oportunidades en la sociedad hiperconectada. Profesorado. Revista de currículum y formación del profesorado, 19(2), 1-16. http://www.ugr.es/local/recfpro/rev192ed.pdf

Fernández, M. (2013). El aprendizaje difuso y el declive de la institución escolar. Revista de la Asociación de Sociología de la Educación (RASE ), 6(2), 150-167. https://doi.org/10.7203/RASE.6.2.8351

Ferreira, S. M., \& Castilho, L. (2018). Aprendizaje ubicuo, interfaces de comunicación y las competencias mediáticas. Universitas, Revista de Ciencias Sociales y Humanas, 29, 201-215. https://doi.org/10.17163/uni.n29.2018.09

García-Sánchez, S., \& Santos-Espino, J. M. (2017). Empowering Pre-Service Teachers to Produce Ubiquitous Flipped Classes. PROFILE Issues in Teachers' Professional Development, 19(1), 169-185. https://doi.org/10.15446/profile.v19n1.53857

García, L. (2017). Educación a distancia y virtual: calidad, disrupción, aprendizajes adaptativo y móvil. RIED. Revista Iberoamericana de Educación a Distancia, 20(2), 9-25. https://doi.org/10.5944/ried.20.2.18737

Gilman, E., Milara, I. S., Cortés, M., \& Riekki, J. (2015). Towards user support in ubiquitous learning systems. IEEE Transactions on Learning Technologies, 8(1), 55-68. https://doi.org/10.1109/TLT.2014.2381467

González-Sanmamed, M., Sangrà, A., Souto-Seijo, A., \& Blanco, I. E. (2018). Ecologías de aprendizaje en la era digital: desafíos para la educación superior. Publicaciones de la Facultad de Educacion y Humanidades del Campus de Melilla, 48(1), 25-45. https://doi.org/10.30827/publicaciones.v48i1.7329

Heredia, J. A. (2016). La incorporación de las TIC y de los dispositivos móviles a la enseñanza de lengua: prácticas y percepciones de los profesores de ELE. El caso de Japón. MarcoELE. Revista de didáctica español lengua extranjera, 22.

Hermann, A. (2013). El entramado sociotécnico en la construcción del conocimiento en la sociedad red. Revista Sophia: Colección de Filosofía de la Educación, 15. https://doi.org/10.17163/soph.n15.2013.09

Huang, Y.-M., \& Chiu, P.-S. (2015). The effectiveness of the meaningful learning-based evaluation for different achieving students in a ubiquitous learning context. $\begin{array}{llll}\text { Computers and } \quad \text { Education, } & \text { 243-253. }\end{array}$ https://doi.org/10.1016/j.compedu.2015.06.009

Huang, Y.-M., Chiu, P.-S., Liu, T.-C., \& Chen, T.-S. (2011). The design and implementation of a meaningful learning-based evaluation method for ubiquitous learning. Computers and Education, 57, 2291-2302. https://doi.org/10.1016/j.compedu.2011.05.023

Huang, Y.-M., Kuo, Y.-H., Lin, Y.-T., \& Cheng, S.-C. (2008). Toward interactive mobile synchronous learning environment with context-awareness service. Computers and Education, 51(3), 1205-1226. https://doi.org/10.1016/j.compedu.2007.11.009

Huang, Y.-M., \& Lin, P. H. (2017). Evaluation students'learning achievement and flow experience with tablet PCs based on AR and tangible technology in u-learning. Library Hi Tech, 32(3), 409-422. https://doi.org/10.1108/LHT-01-2017-0023

Análisis crítico del concepto "aprendizaje ubicuo" a través de la Cartografía Conceptual. Belén Velázquez Gatica y Rocío Edith López Martínez. 
Humanante-Ramos, P., García-Peñalvo, F. J., \& Conde-González, M. (2017). Entornos personales de aprendizaje móvil: una revisión sistemática de la literatura. RIED. Revista Iberoamericana de Educación a Distancia, 20(2), 73-92. https://doi.org/10.5944/ried.20.2.17692

Hwang, G.-J., Hung, P.-H., Chen, N.-S., \& Liu, G.-Z. (2014). Mindtool-assisted in-field learning (MAIL): An advanced ubiquitous learning project in Taiwan. Educational Technology and Society, 17(2), 4-16.

Hwang, G.-J., Yang, T.-C., Tsai, C.-C., \& Yang, S. J. H. (2009). A context-aware ubiquitous learning environment for conducting complex science experiments. Computers and Education, 53(2), 402-413. https://doi.org/10.1016/j.compedu.2009.02.016

Hwang, G.-J., Zou, D., \& Lin, J. (2020). Effects of a multi-level concept mapping-based question-posing approach on students' ubiquitous learning performance and perceptions. Computers and Education, 149(January). https://doi.org/10.1016/j.compedu.2020.103815

Hwang, G. J., Shi, Y. R., \& Chu, H. C. (2011). A concept map approach to developing collaborative Mindtools for context-aware ubiquitous learning. British Journal of Educational Technology, 42(5), 778-789. https://doi.org/10.1111/j.14678535.2010.01102.x

Islas, C., \& Carranza, M. R. (2017). Ecosistemas digitales y su manifestación en el aprendizaje: Análisis de la literatura. Revista de Educación a Distancia (RED), 55. https://doi.org/10.6018/red/55/9

Jung, H.-J. (2014). Ubiquitous learning: Determinants impacting learners' satisfaction and performance with smartphones. Language, Learning and Technology, 18(3), 97-119. http://lit.msu.edu/issues/october2014/jung.pdf

Karchoud, R., Roose, P., Dalmau, M., Illarramendi, A., \& Ilarri, S. (2017). All for One and One for All: Dynamic Injection of Situations in a Generic Context-Aware Application. Procedia Computer Science, 113, 17-24. https://doi.org/10.1016/j.procs.2017.08.277

Karimi, S. (2016). Do learners' characteristics matter? An exploration of mobile-learning adoption in self-directed learning. Computers in Human Behavior, 63, 769-776. https://doi.org/10.1016/j.chb.2016.06.014

Kim, H.-J., Caytiles, R. D., \& Kim, T.-H. (2012). Design of an effective WSN-based interactive u-learning model. International Journal of Distributed Sensor Networks, 2012. https://doi.org/10.1155/2012/514836

Kong, J. (2019). Innovative applications mode of network learning space in exercise physiology based on ubiquitous learning. International Journal of Emerging Technologies in Learning, 14(4), 113-126. https://doi.org/10.3991/ijet.v14i04.10117

López, L. R. (2017). Indagación en la relación entre aprendizaje - tecnologías digitales. Educación y Educadores, 20(1), 91-105. https://doi.org/10.5294/edu.2017.20.1.5

Lucke, U., \& Rensing, C. (2013). A survey on pervasive education. Pervasive and Mobile 
Computing, 14, 1-14. https://doi.org/10.1016/j.pmcj.2013.12.001

Manolakis, L. (2011). Ubiquitous learning. Propuesta Educativa, 1(35), 123-126.

Marín, J. G. (2016). Enseñanza del lenguaje y nuevos alfabetismos; entre la tradición y la innovación. IE Revista de investigación educativa de la REDIECH, 7(13), 21-33.

Marinagi, C., Skourlas, C., \& Belsis, P. (2013). Employing ubiquitous computing devices and technologies in the higher education classroom of the future. Procedia - Social and Behavioral Sciences, 73, 487-494. https://doi.org/10.1016/j.sbspro.2013.02.081

Martinez-Pabon, F., Ramires-Gonzalez, G., \& Chantre-Astaiza, Á. (2014). Modelo conceptual para el despliegue de publicidad ubicua soportado en un esquema de cooperación Smart TV - Smartphone. Ciencia e Ingeniería Neogranadina, 24(1), 116-142.

Mojarro, Á., Rodrigo, D., \& Etchegaray, M. C. (2015). Educación personalizada a través de e-Learning. Alteridad. Revista de Educacion, 10(1), 21-30. https://doi.org/10.17163/alt.v10n1.2015.02

Moreno-López, G. A., Ramírez-Monsalve, E. de J., \& Jiménez-Builes, J. A. (2017). Ubiquitous learning model based on platforms of multi-screen TV (uLMTV). Dyna, 84(203), 160-169. https://doi.org/10.15446/dyna.v84n203.64160

Moreno, G. A., Jiménez, J. A., \& Bernal, S. C. (2016). Overview of u-learning. Concepts, characteristics, uses, application scenarios and topics for research. IEEE Latin America Transactions, 14(12), 4792-4798. https://doi.org/10.1109/TLA.2016.7817013

Oliveira, R., \& Horta, G. (2012). Towards a framework to characterize ubiquitous software projects. Information and Software Technology, 54(7), 759-785. https://doi.org/10.1016/j.infsof.2012.01.009

Ortega-Carbajal, M. F., Hernández-Mosqueda, J. S., \& Tobón-Tobón, S. (2015). Impacto de la cartografía conceptual como estrategia de gestión del conocimiento. $R a$ Ximhai, 11(4), 171-180.

Osman, H. M., Singh, M. M., Shariff, A. R. M., Bakar, A. A., \& Plasencia, M. S. (2018). Enhanced Analytical Hierarchy process for U-Learning with Near Field Communication (NFC) technology. International Journal of Advanced Computer Science and Applications, 9(12), 281-290. https://doi.org/10.14569/IJACSA.2018.091241

Páramo, P., Hederich, C., López, O., Sanabria, L. B., \& Camargo, Á. (2015). ¿Dónde ocurre el aprendizaje? Psicogente, 18(34), 320-335. https://doi.org/10.17081/psico.18.34.508

Park, Y. (2011). A pedagogical framework for mobile learning: Categorizing educational applications of mobile technologies into four types. International Review of Research in Open and Distance Learning, 12(2), 78-102. https://doi.org/10.19173/irrodl.v12i2.791

Pimmer, C., Mateescu, M., \& Gröhbiel, U. (2016). Mobile and ubiquitous learning in higher education settings. A systematic review of empirical studies. Computers in

Análisis crítico del concepto "aprendizaje ubicuo" a través de la Cartografía Conceptual. Belén 
Human Behavior, 63, 490-501. https://doi.org/10.1016/j.chb.2016.05.057

Pradeep, P., \& Krishnamoorthy, S. (2019). The MOM of context-aware systems: A survey. Computer Communications, 137(January), 44-69. https://doi.org/10.1016/j.comcom.2019.02.002

Quintero, S. P., Díaz, Á. M., \& Ortiz, G. E. (2015). Modelo de mediaciones pedagógicas y tecnológicas para entender e incorporar adecuadamente procesos pedagógicoscomunicativos-tecnológicos de ambientes virtuales de aprendizaje en la Policia Nacional de Colombia. Revista Logos, Ciencia \& Tecnología, 6(2), 188-197.

Ramirez, G. M., Collazos, C. A., \& Moreira, F. (2017). All-Learning: The state of the art of the models and the methodologies educational with ICT. Telematics and Informatics, 35(4), 944-953. https://doi.org/10.1016/j.tele.2017.10.004

Ranga, J. S. (2018). Multipurpose Use of Explain Everything iPad App for Teaching Chemistry Courses. Journal of Chemical Education, 95(5), 895-898. https://doi.org/10.1021/acs.jchemed.7b00676

Raza, S., \& Ding, C. (2019). Progress in context-aware recommender systems - An overview. Computer Science Review, 31, 84-97. https://doi.org/10.1016/j.cosrev.2019.01.001

Real Academia Española. (2014). Diccionario de la lengua española (Versión 23). https://dle.rae.es

Rodrigo, Miguel, Á., \& de Castro, C. (2013). La información digital actual, un nuevo modelo de contenido educativo para un entorno de aprendizaje ubicuo. RED. Revista de , 39. http://www.um.es/ead/red/39/

Sevillano-García, M. L., González-Flores, M. del P., Vázquez-Cano, E., \& Yedra, L. (2016). Ubicuidad y movilidad de herramientas virtuales abren nuevas expectativas formativas para el estudiantado universitario. Revista Ensayos Pedagógicos, 11(2), 99-131.

Sobrino, Á. (2011). Proceso de enseñanza-aprendizaje y web 2.0: valoración del conectivismo como teoría de aprendizaje post-constructivista. Estudios sobre educacion, 20, 117-140.

Souabni, R., Saâdi, I. B., Kinshuk, \& Ghezala, H. Ben. (2017). Context Weighting for Ubiquitous Learning Situation Description: Approach Based on Combination of Weighted Experts' Opinions. International Journal of Information Technology and Decision Making, 16(1), 247-309. https://doi.org/10.1142/S0219622017500407

Tobón-Tobón, S. (2004). Estrategias didácticas para formar competencias. Módulo V. La cartografía conceptual. IV Congreso Internacional Virtual de Educación. https://www.cife.edu.mx/Biblioteca/public/Libros/7/Cartografiaconceptual2005Esp ana.pdf

Tobón-Tobón, S. (2012). Cartografía conceptual: estrategia para la formación y evaluación de conceptos y teorías. Instituto CIFE.

Tobón, S., Gonzalez, L., Nambo, J. S., \& Vazquez, J. M. (2015). La Socioformación: Un Estudio Conceptual. XXadigma, XXVI(1), 7-29.

Análisis crítico del concepto "aprendizaje ubicuo" a través de la Cartografía Conceptual. Belén

Velázquez Gatica y Rocío Edith López Martínez. 
http://revistas.upel.edu.ve/index.php/paradigma/article/view/2661/1273

Uribe-Zapata, A. (2018). Concepto y prácticas de educación expandida: una revisión de la literatura académica. El Ágora U.S.B., 18(1), 278-293. https://doi.org/10.21500/16578031.3456

Uribe, A. (2017). Una revisión de prácticas educativas expandidas de la ciudad de Medellín. Revista científica Guillermo de Ockham, 15(1), 14. https://doi.org/10.21500/22563202.2757

Valenzuela-Valdés, J. F., Pardo, P. J., Padilla, P., \& Lozano-Guerrero, A. J. (2016). Low Cost Ubiquitous Context-Aware Wireless Communications Laboratory for Undergraduate Students. IEEE Transactions on Learning Technologies, 9(1), 3136. https://doi.org/10.1109/TLT.2015.2438864

Victória, J. L., Martins, C., Kerber, L., \& Ferrari, D. N. (2016). TrailTrade: A model for trail-aware commerce support. Computers in Industry, 80, 43-53. https://doi.org/10.1016/j.compind.2016.04.006

Vinu, P. V., Sherimon, P. C., \& Krishnan, R. (2011). Towards pervasive mobile learning - the vision of 21st century. Procedia Social and Behavioral Sciences, 15, 30673073. https://doi.org/10.1016/j.sbspro.2011.04.247

Wagner, A., Victória, J. L., \& Ferrari, D. N. (2014). A model for profile management applied to ubiquitous learning environments. Expert Systems with Applications, 41, 2023-2034. https://doi.org/10.1016/j.eswa.2013.08.098

Wang, S.-L., \& Wu, C.-Y. (2011). Application of context-aware and personalized recommendation to implement an adaptive ubiquitous learning system. Expert Systems with Applications, 38(9), 10831-10838. https://doi.org/10.1016/j.eswa.2011.02.083

Weiser, M. (1991). The Computer for the 21st Century. Scientific American Ubicomp, 265(3), 94-104.

Wen, C., \& Zhang, J. (2014). Design of a Microlecture Mobile Learning System Based on Smartphone and Web Platforms. IEEE Transactions on Education, 58(3), 203207. https://doi.org/10.1109/TE.2014.2363627

Wong, L.-H., \& Looi, C.-K. (2011). What seams do we remove in mobile-assisted seamless learning? A critical review of the literature. Computers and Education, 57, 2364-2381. https://doi.org/10.1016/j.compedu.2011.06.007

Xie, H., Chu, H.-C., Hwang, G.-J., \& Wang, C.-C. (2019). Trends and development in technology-enhanced adaptive/personalized learning: A systematic review of journal publications from 2007 to 2017. Computers and Education, 140, 1-16. https://doi.org/10.1016/j.compedu.2019.103599

Yang, C.-C., Hwang, G.-J., Hung, C.-M., \& Tseng, S.-S. (2013). An evaluation of the learning effectiveness of concept map-based science book reading via mobile devices. Educational Technology and Society, 16(3), 167-178.

Zapata-Ros, M. (2015a). Calidad en entornos ubicuos de aprendizaje. RED-Revista de Educación a Distancia, 31. https://revistas.um.es/red/article/view/232871

Análisis crítico del concepto "aprendizaje ubicuo" a través de la Cartografía Conceptual. Belén Velázquez Gatica y Rocío Edith López Martínez. 
Zapata-Ros, M. (2015b). Teorías y modelos sobre el aprendizaje en entornos conectados y ubicuos: Bases para un nuevo modelo teórico a partir de una visión crítica del "conectivismo". Education in the Knowledge Society, 16(1), 69-102. https://doi.org/10.14201/eks201516169102 